\title{
Non-equilibrium condensation of water vapour in supersonic flows
}

with shock waves

Chuang Wen ${ }^{1,2}$, Nikolas Karvounis ${ }^{1}$, Jens Honore Walther ${ }^{1,3}$, Hongbing Ding ${ }^{4}$, Yan Yang $^{1, *}$

${ }^{1}$ Department of Mechanical Engineering, Technical University of Denmark, Nils Koppels Allé, 2800 Kgs. Lyngby, Denmark

${ }^{2}$ Faculty of Engineering, University of Nottingham, University Park, Nottingham NG7 2RD, UK

${ }^{3}$ Computational Science and Engineering Laboratory, ETH Zürich, Clausiusstrasse 33, CH-8092 Zürich, Switzerland

${ }^{4}$ Tianjin Key Laboratory of Process Measurement and Control, School of Electrical and Information Engineering, Tianjin University, Tianjin 300072, China

*Corresponding author: Yan Yang, Email: yyang@mek.dtu.dk

Abstract: The fluid flow and heat and mass transfer in a supersonic separator are not understood well due to the complicated interaction of the supersonic flow, swirling flow, phase transition and shock waves. In the present study, we develop a wet steam model to investigate the flow structure inside a supersonic separator with the co-existence of non-equilibrium condensation and shock waves. A study of the effect of the inlet subcooling and inlet saturation on the condensation behaviour is conducted to evaluate the performance of the supersonic separation with a focus on the shock wave. The numerical result shows that the degree of supersaturation of the water vapour can reach a maximum value of 4.28 within the designed supersonic separator and generate a peak 
nucleation rate of approximately $10^{21} \mathrm{~kg} \mathrm{~m}^{-3} \mathrm{~s}^{-1}$. The occurrence of the shock wave changes the equilibrium thermodynamic state, which leads to the re-evaporation of the condensed droplet. Higher inlet subcooling and inlet saturation not only shift downstream the position of the shock wave, but also induce an earlier condensation and higher liquid fraction. For the present nozzle, when the inlet subcooling and inlet saturation are about $34 \mathrm{~K}$ and 0.28 respectively, the shock wave intersects the region of the intense nucleation process, the non-equilibrium condensation process is terminated due to the increase of the pressure and temperature downstream the shock wave. Stronger swirling flow results in non-uniform distribution of the static pressure and decreases the nucleation rate of water vapour. The high swirling flow with a maximum swirl velocity of $150 \mathrm{~m} / \mathrm{s}$ weakens the liquid fraction by $25 \%$ compared to the no swirling flow. This indicates that it is important to balance the swirling flow and condensation process to achieve an efficient performance of the supersonic separator.

Keywords: wet steam; condensing flow; supersonic flow; condensation; shock wave; swirling flow

\section{Introduction}

The supersonic separator has been introduced for natural gas processing with a focus on the removal of water vapour[1,2]. The coexistence of non-equilibrium condensation and a shock wave in supersonic flows is a major feature of the separator. On the one hand, the key working principle of the supersonic separation is nonequilibrium condensation of water vapour in supersonic flows. On the other hand, the supersonic separation also benefits from the shock wave [3] when supersonic flows are 
transformed to subsonic flows, which recovers the pressure to maximum improve the energy efficiency [4].

Non-equilibrium condensation in a supersonic nozzle has been investigated for several decades without considering a shock wave [5-7]. Dykas \& Wróblewski [8] developed the single-fluid and two-fluid wet steam model to calculate the condensation flow in a Laval nozzle and it was found that the single-fluid model underestimated the droplet diameters by approximately $40 \%$ compared to the two-fluid model. Abadi et al. [9] employed a two-fluid wet steam model to study the condensing flow inside a highpressure nozzle, observing a slip velocity and temperature difference between liquid and vapour phases were. Pillai \& Prasad [10] evaluated the influence of wall surface roughness on non-equilibrium condensation in a Laval nozzle using a wet steam model, and the numerical result indicated that the boundary layer thickness increased by $33 \%$ while the roughness height varied from $1 \mu \mathrm{m}$ to $1000 \mu \mathrm{m}$. Zhao et al. [11] numerically investigated the effect of the converging profile on non-equilibrium condensation processes in a supersonic nozzle, and the results showed that a slow converging section of the supersonic nozzle delayed the occurrence of the condensation position.

The wet steam in turbine blade cascades is another research focus for nonequilibrium condensation in supersonic flows, in which the shock wave exists but is not specifically investigated [12-14]. Wróblewski et al. [15] developed and validated a single-fluid wet steam model for the calculation of condensation flows in turbine cascades. They found that the calculated droplet size was smaller than those in experiments for low inlet pressures and higher for high inlet pressures. Vatanmakan et 
al. [16] studied the entropy generation in condensing flow in turbine blades and the numerical result showed that including volumetric heating to the convergent section reduced the liquid fraction. Ding et al. [17] evaluated the effect of surface roughness on condensing flow in turbine blades using the single-fluid wet steam model. Their results suggested that the liquid fraction at the outlet slightly decreased with the roughness height.

For supersonic separators, the working fluid was mainly assumed as a single-phase gas without considering the phase transition process [18], while only a few studies involved the condensation phenomenon. Ma et al. [19] established a two-fluid flow model to investigate the spontaneous condensation of water vapour using an ideal gas equation of state for the calculation of thermodynamic properties. They employed the model to evaluate the influence of the external particle on the droplet condensation and their numerical results indicated that it was reasonable to add external nuclei to increase the size of the condensed droplet [20]. Shooshtari and Shahsavand [21, 22] developed a one-dimensional condensing flow model to calculate condensation rates in supersonic separators, and their computational results agreed well with process simulation results and experimental data. Based on the CFD model in [21, 22], they investigated the effect of heterogeneous condensation processes on the removal of water vapour inside a supersonic separator, and the optimization analysis showed that the injection of $2.4 \%$ solid particles of $2 \mu \mathrm{m}$ diameter provided the best separation performance [23]. In the aforementioned studies, the nucleation and condensation of water vapour in a supersonic separator was numerically calculated with the ideal gas model $[19,20]$ or 
one-dimensional flow model [21-23].

The purpose of this study is to evaluate the fluid flow inside a supersonic separator with the coexistence of non-equilibrium condensation and shock waves. Since the aforementioned studies have not specifically focus on the shock wave, they did not fully capture relevant aspects of the wet steam flow. The key contribution of this work can be summarized as a) development and validation of a computational fluid dynamics modelling that captures the condensation and shock wave in supersonic flows, b) effect of inlet conditions on the condensation process considering the shock wave, c) influence of the shock wave on the condensation process, d) investigation of the role of the swirling flow on the condensation process in supersonic flows.

\section{Supersonic separators}

A supersonic separator usually consists of four parts, a set of static vanes, a converging-diverging nozzle, a collector and a diffuser, as shown in Fig. 1. In the converging-diverging nozzle, the fluid flow is accelerated from subsonic to supersonic, in which the smooth transition between the converging part and the diverging part plays a significant role to avoid the disturbance by oblique shock waves [24]. For this purpose, the profile of the converging part of the nozzle is described by Eq. (1), which provides a curve continuity to the diverging part of the nozzle.

$$
\begin{aligned}
& \frac{D-D_{t h}}{D_{i n}-D_{t h}}=1-\frac{1}{a^{2}}\left(\frac{x}{L_{1}}\right)^{3} \quad\left(\frac{x}{L_{1}} \leq a\right) \\
& \frac{D-D_{t h}}{D_{i n}-D_{t h}}=\frac{1}{(1-a)^{2}}\left(1-\frac{x}{L_{1}}\right)^{3} \quad\left(\frac{x}{L_{1}}>a\right)
\end{aligned}
$$

where $D_{i n}, D_{t h}$ and $D$ are the diameter of the nozzle at the inlet, throat and $x$, respectively. 
$L_{1}$ is the length of the converging part of the nozzle. $a$ is the design coefficient with a value of 0.5 for this study.

Two-dimensional simulations are performed neglecting the static vanes as the focus of the present study is non-equilibrium condensation phenomenon. In this specific design, the diverging part of the nozzle is extended with a length of $200 \mathrm{~mm}$, which simultaneously undertakes two functions of refrigeration and cyclonic separation. The diameters at the throat and the outlet of the converging-diverging nozzle are $17.40 \mathrm{~mm}$ and $21.40 \mathrm{~mm}$, respectively. The lengths of the converging part of the nozzle and the diffuser are $186.60 \mathrm{~mm}$ and $300 \mathrm{~mm}$, respectively. In addition, a constant pipe with a length of $200 \mathrm{~mm}$ is installed upstream and downstream of the supersonic separator. Table 1 lists the detailed dimensions of the designed supersonic separator. The specified equipment size is compatible with a lab-scale unit.

The structured mesh is generated for the designed supersonic separator without considering the static vanes, and the details of the grid system are shown in Fig. 2. The grid independence study is carried out to analyse the effect of the grid resolution on the prediction of non-equilibrium condensation in supersonic separators. Three different grid sizes are tested based on a coarse mesh of 20000 cells, a medium mesh of 55580 cells and a fine mesh of 287051 cells. The flow structure and condensation properties are utilised as the characteristic parameters for the grid independence study including the static pressure and the nucleation rate.

Figure 3 describes the effect of the grid size on the static pressure and nucleation rate inside the supersonic separator. The results predicted by the coarse mesh are 
131

132

different from the other two cases both on the distributions of the static pressure and nucleation rate, in particular, the appearance of the shock wave for the coarse mesh case.

This means that the coarse mesh can hardly capture the shock wave in supersonic flows.

The medium mesh and fine mesh, on the contrary, predict similar static pressure and nucleation rate. Therefore, the medium mesh of 55580 cells is employed for the numerical simulation of non-equilibrium condensation in supersonic separators considering the numerical accuracy and computational cost.

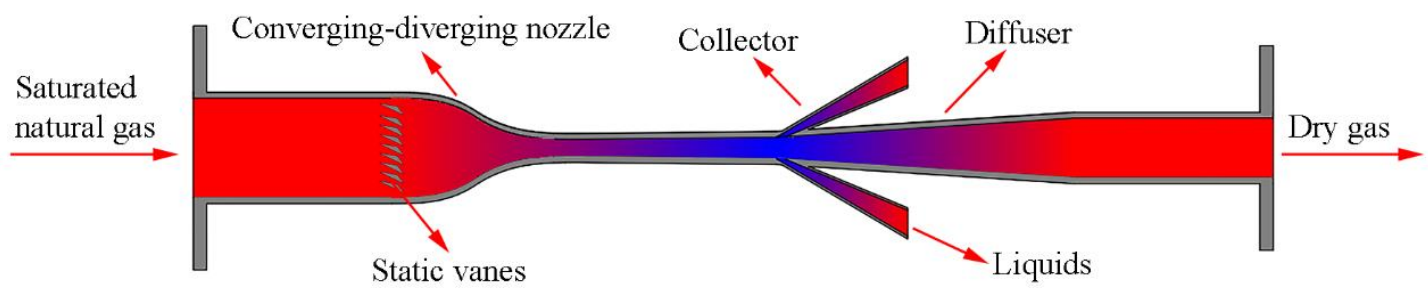

Fig 1. Schematic of a supersonic separator including static vanes (not considered in the present study); converging-diverging nozzle, collector (not considered in the present study) and diffuser.

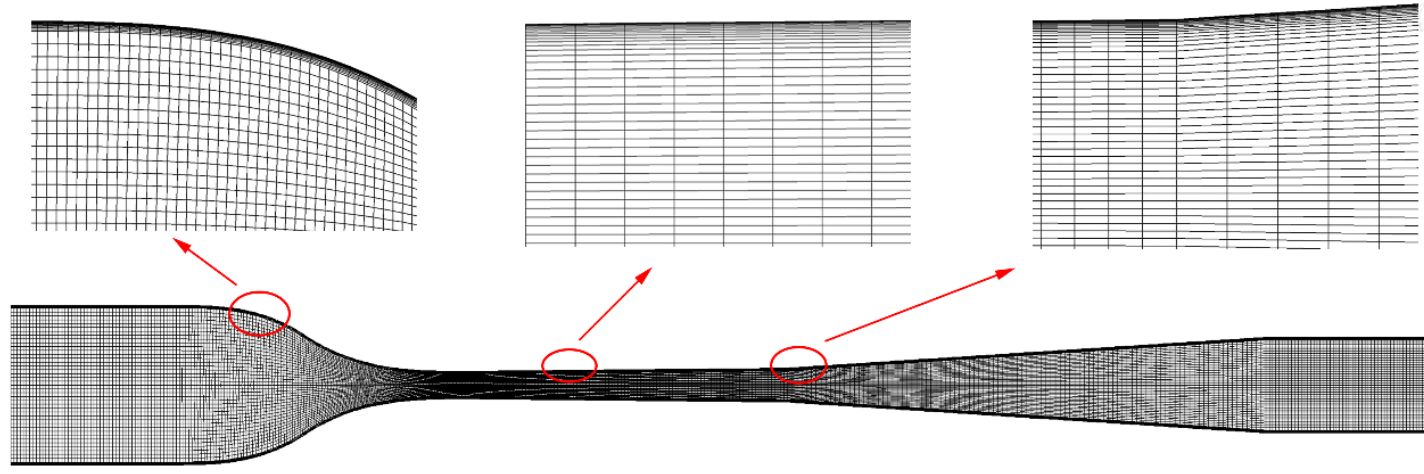

Fig 2. Structured finite-volume mesh of a supersonic separator. Table 1. Dimensions of the designed supersonic separator $\begin{array}{ll}\text { Geometrical parameters } & \text { Value }(\mathrm{mm})\end{array}$

Inlet diameter of the converging-diverging nozzle $\left(D_{i n}\right) \quad 100.00$ 
Throat diameter of the converging-diverging nozzle $\left(D_{t h}\right)$

Outlet diameter of the converging-diverging nozzle $\left(D_{\text {out }}\right) \quad 21.40$

Length of the converging part of the nozzle $\left(L_{n c}\right) \quad 186.60$

Length of the diverging part of the nozzle $\left(L_{n d}\right) \quad 200.00$

Outlet diameter of the diffuser $\left(D_{d i}\right) \quad 60.00$

Length of the diffuser $\left(L_{d i}\right) \quad 300.00$

Length of the constant tube $\left(L_{c t}\right) \quad 200.00$

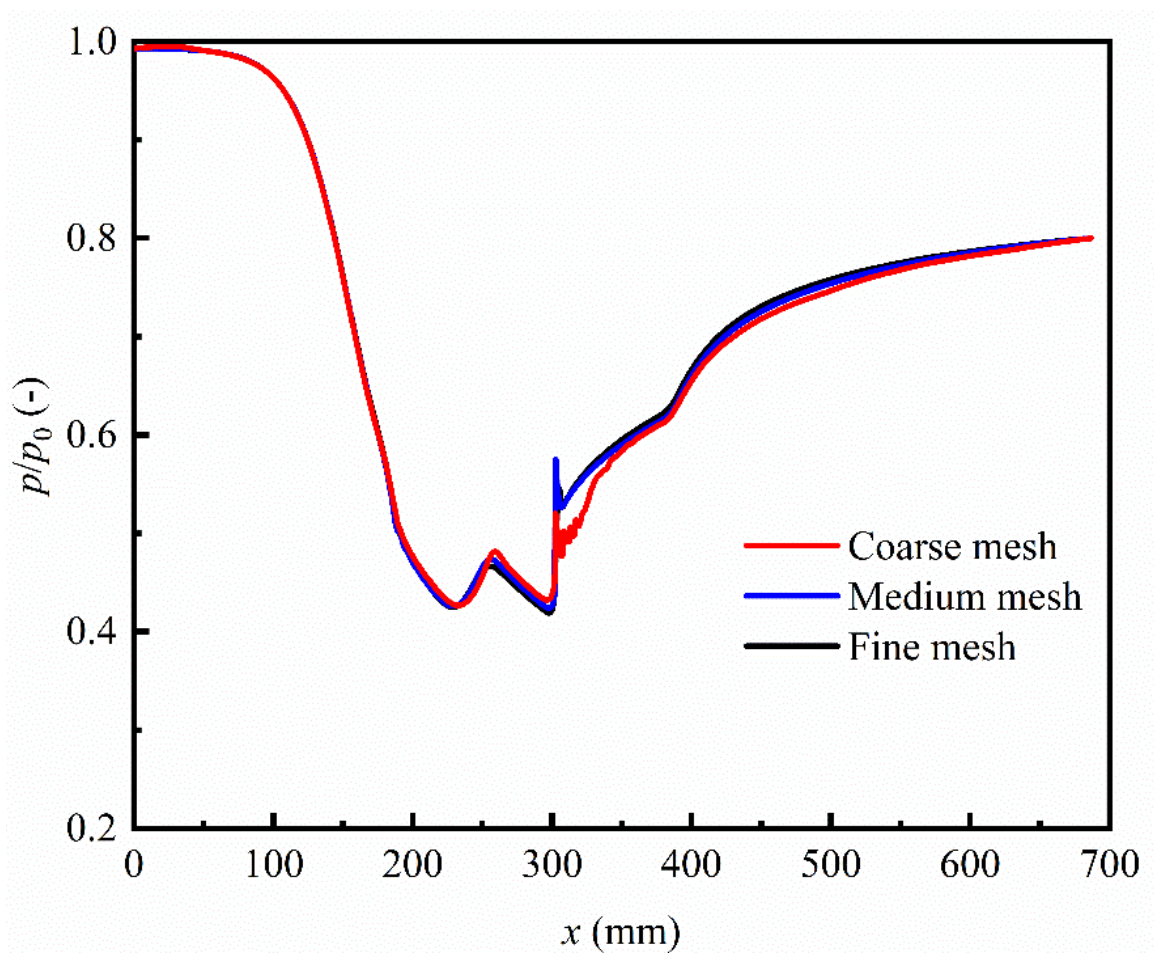




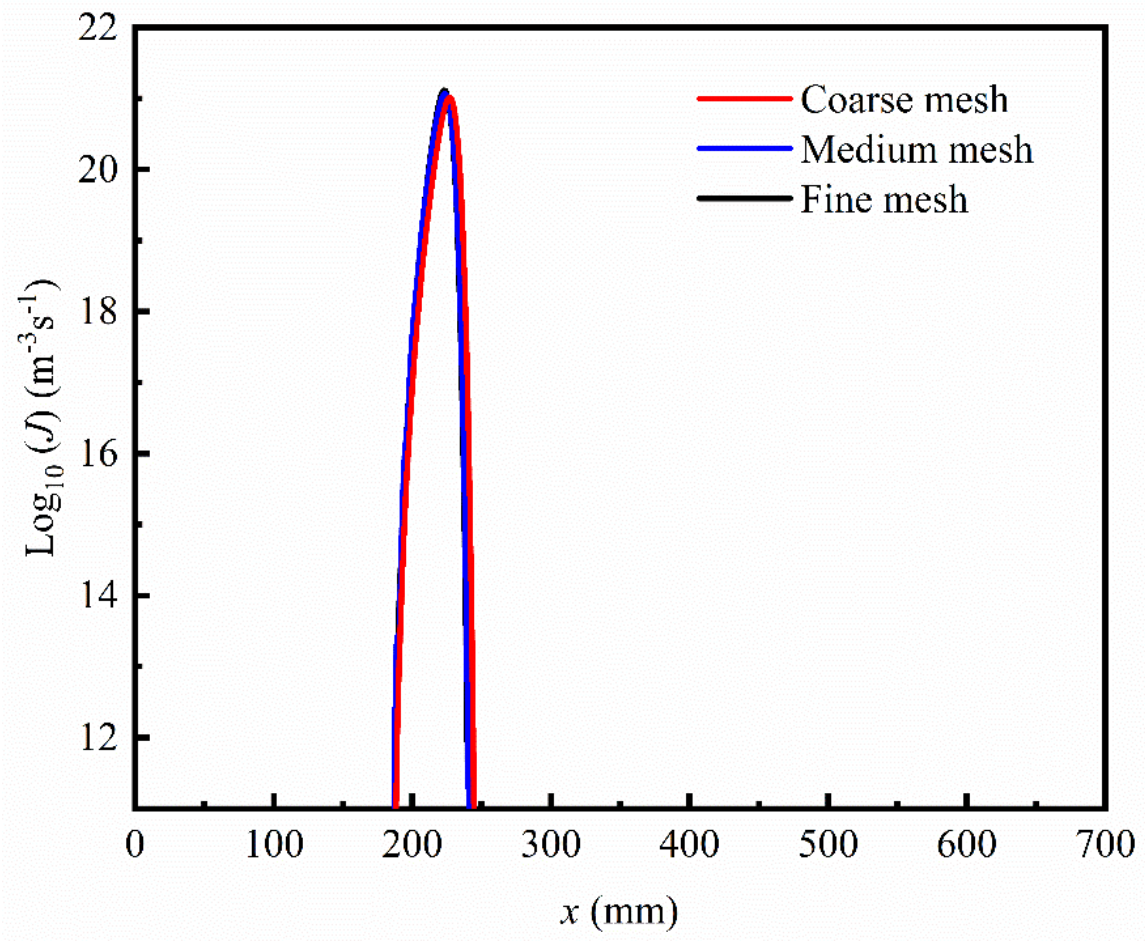

147

148

149

150

151

152

153

154

155

156

157

158

159

160

(b)

Fig. 3 Effect of grid resolution on the static pressure (a) and nucleation rate (b) in a supersonic separator

\section{Mathematical model}

\subsection{Wet steam model}

The compressible Navier-Stokes equations are used to describe the nonequilibrium condensation under supersonic conditions. The phase change model, including the nucleation and droplet growth processes $[25,26]$, is established to predict the condensation process. The assumption of no-slip velocity between the vapour phase and the liquid phase is adopted in our CFD modelling as the droplet size is small, i.e., usually less than $1 \mu \mathrm{m}[27,28]$. The model equations are:

Equation of continuity:

$$
\frac{\partial \rho}{\partial t}+\frac{\partial\left(\rho u_{j}\right)}{\partial x_{j}}=-\Gamma
$$




$$
\frac{\partial}{\partial t}\left(\rho u_{i}\right)+\frac{\partial}{\partial x_{j}}\left(\rho u_{j} u_{i}\right)=-\frac{\partial p}{\partial x_{i}}+\frac{\partial \tau_{i j}}{\partial x_{j}}-u_{i} \Gamma
$$

Equation of energy:

$$
\begin{aligned}
\frac{\partial}{\partial t}(\rho H)+\frac{\partial}{\partial x_{j}}\left(\rho u_{j} H+p\right) & =-\frac{\partial}{\partial x_{j}}\left(\lambda_{e f f} \frac{\partial T}{\partial x_{j}}\right)+\frac{\partial}{\partial x_{j}}\left(u_{i} \tau_{i j}\right)-h_{l v} \Gamma \\
H & =C_{p} T+\frac{1}{2} u_{i} u_{i}
\end{aligned}
$$

where $\rho, u_{i}, p, T$ and $H$ are the density, velocity, pressure, temperature and total specific enthalpy, respectively. $h_{l v}$ is the latent heat. $C_{p}$ is the constant pressure specific heat. $\lambda_{e f f}$ is the effective conductivity, $\lambda_{\text {eff }}=\lambda_{v}+\lambda_{t}$, where $\lambda_{v}$ and $\lambda_{t}$ are the molecular conductivity and turbulent thermal conductivity, respectively. $\tau_{i j}$ is viscous stress, and the source term $\Gamma$ describes the condensation mass rate of the water vapour.

Two transport equations are utilized to describe the phase change process during non-equilibrium condensation. These include the liquid fraction $(Y)$ and droplet number per volume $(N)[29]$ :

$$
\begin{aligned}
& \frac{\partial(\rho Y)}{\partial t}+\frac{\partial}{\partial x_{j}}\left(\rho Y u_{j}\right)=\Gamma \\
& \frac{\partial(\rho N)}{\partial t}+\frac{\partial}{\partial x_{j}}\left(\rho N u_{j}\right)=\rho J
\end{aligned}
$$

where $J$ is the nucleation rate. $N$ is the number of droplets per volume. $\Gamma$ is the condensation mass rate per unit vapour volume per unit time [26]:

$$
\Gamma=\frac{4 \pi r_{c}^{3}}{3} \rho_{l} J+4 \pi r^{2} \rho_{l} N \frac{d r}{d t}
$$

where $\rho_{l}$ is the droplet density, $r$ is the droplet radius. $d r / d t$ is the growth rate of droplets, and $r_{c}$ is the Kelvin-Helmholtz critical droplet radius. 

which uses the non-isothermal correction of Kantrowitz [30] as follows:

$$
J=\frac{q_{c}}{1+\phi} \frac{\rho_{v}^{2}}{\rho_{l}} \sqrt{\frac{2 \sigma}{\pi m_{v}{ }^{3}}} \exp \left(-\frac{4 \pi \sigma}{3 k_{B} T_{v}} r_{c}^{2}\right)
$$

where $q_{c}$ is the condensation coefficient ( $q_{c}=1.0$ in this study), $\sigma$ is the liquid surface tension, $m_{v}$ is the mass of a vapour molecule, $k_{B}$ is the Boltzmann's constant. $T_{v}$ is the vapour temperature, which is obtained from Eqs (4) and (5). $\phi$ is a correction factor proposed by Kantrowitz [30]:

$$
\phi=2 \frac{\gamma-1}{\gamma+1} \frac{h_{l v}}{R_{v} T_{v}}\left(\frac{h_{l v}}{R_{v} T_{v}}-\frac{1}{2}\right)
$$

where $\gamma$ is the specific heat ratio, and $R_{v}$ is the gas constant.

The growth rate of droplets due to evaporation and condensation, $d r / d t$, is calculated by Young's model [31]

$$
\frac{d r}{d t}=\frac{\lambda_{v}\left(T_{s}-T_{v}\right)}{\rho_{l} h_{l v} r} \frac{\left(1-r_{c} / r\right)}{\left(\frac{1}{1+2 \beta \mathrm{Kn}}+3.78(1-v) \frac{\mathrm{Kn}}{\mathrm{Pr}}\right)}
$$

where $T_{s}$ is the saturated temperature, Pr is the Prandtl number, Kn is the Knudsen number and $v$ is the modelling correction coefficient:

$$
v=\frac{R_{v} T_{s}}{h_{l v}}\left(\alpha-0.5-\frac{2-q_{c}}{2 q_{c}}\left(\frac{\gamma+1}{2 \gamma}\right)\left(\frac{C_{p} T_{s}}{h_{l v}}\right)\right)
$$

where $\alpha$ and $\beta$ are the modelling parameters ( $\alpha=1.0$ and $\beta=0.0$ in this study).

The ANSYS FLUENT 18 [32] is employed to solve the continuity, momentum and energy conservation equations, while additional two scalar equations are embedded in FLUENT to describe the condensation and droplet growth processes using the UserDefined-Scalar (UDS) and User-Defined-Function (UDF) interfaces. The governing 
equations are discretized by the finite volume method, while the implicit density-based solver is utilized for supersonic flows. The second-order upwind scheme is adopted for an accurate spatial discretization. The pressure inlet and pressure outlet conditions are used for the entrance and exit of the supersonic separator, while no-slip and adiabatic conditions are assumed for the wall boundaries. The $k$ - $\omega$ shear stress transport (SST) turbulence model [33] is used due to the good accuracy to predict supersonic flows [34] and non-equilibrium condensation phenomenon $[35,36]$.

\subsection{Model Validation}

The case of G37-B of the 2/M nozzle in Hedbäck experiments [37] is employed to validate the developed wet steam model for non-equilibrium condensation in supersonic flows. Figure 4 (a) describes the geometry and main dimensions of the supersonic nozzle. The height at the nozzle throat is $10.00 \mathrm{~mm}$, while the heights at the nozzle inlet and outlet are 19.99 and $17.91 \mathrm{~mm}$, respectively. The total pressure and total temperature at the nozzle inlet are $13.83 \mathrm{MPa}$ and $674.42 \mathrm{~K}$ in the experiment, while the nozzle out is the supersonic flow. Figure 4 (b) describes the computational mesh in our numerical studies, and the grid is refined in the near-wall region to ensure that the wall $y+<1$ for the $k-\omega$ SST turbulence model. Before the model validation, the effect of the grid resolution on the flow structure and condensation process is investigated based on the coarse mesh of 22400 cells, the medium mesh of 56400 cells and the fine mesh of 122400 cells. Figure 5 depicts the contours and profiles of the Mach number inside the Hedbäck's nozzle, and Figure 6 represents the nucleation rate in the supersonic nozzle. It can be seen that these three meshes predict almost the same 
Mach number upstream the condensation process. The coarse mesh predicts a tiny higher Mach number than the medium and fine meshes when the steam condensation occurs, while the latter two meshes compute a similar Mach number with deviations less than $0.25 \%$. The profiles of the nucleation rate illustrate that these three different resolutions of the quadrilateral mesh calculate uniform condensation behaviour. However, the medium and fine meshes describe more details of the edge of the nucleation region due to higher grid resolution, when looking into the contours of the nucleation rate. Thus, the medium mesh of 56400 cells is employed for the model validation considering the numerical accuracy and computational costs.

For the model validation, the numerical simulation of the single-phase flow without considering the phase change process is carried out as a basis to clearly show the effect of the condensation process on the flow structure due to the heat and mass transfer between the vapour and liquid phases. The comparison results between the numerical simulation and experimental data for the Hedbäck nozzle [37] are shown in Fig. 7, including the contours and profiles of the static pressure and droplet radius. We can see that our developed wet steam model accurately captures the onset of the condensation process and the static pressure deviates from the isentropic flow. The droplet radius from the numerical simulation also agrees well with experimental data [37]. Thus, our developed wet steam model can be applied for the prediction of the nonequilibrium condensation in supersonic flows from the view of the static pressure and droplet radius. 



Fig. 4 Geometry (a) and mesh (b) for the Hedbäck's nozzle [37]

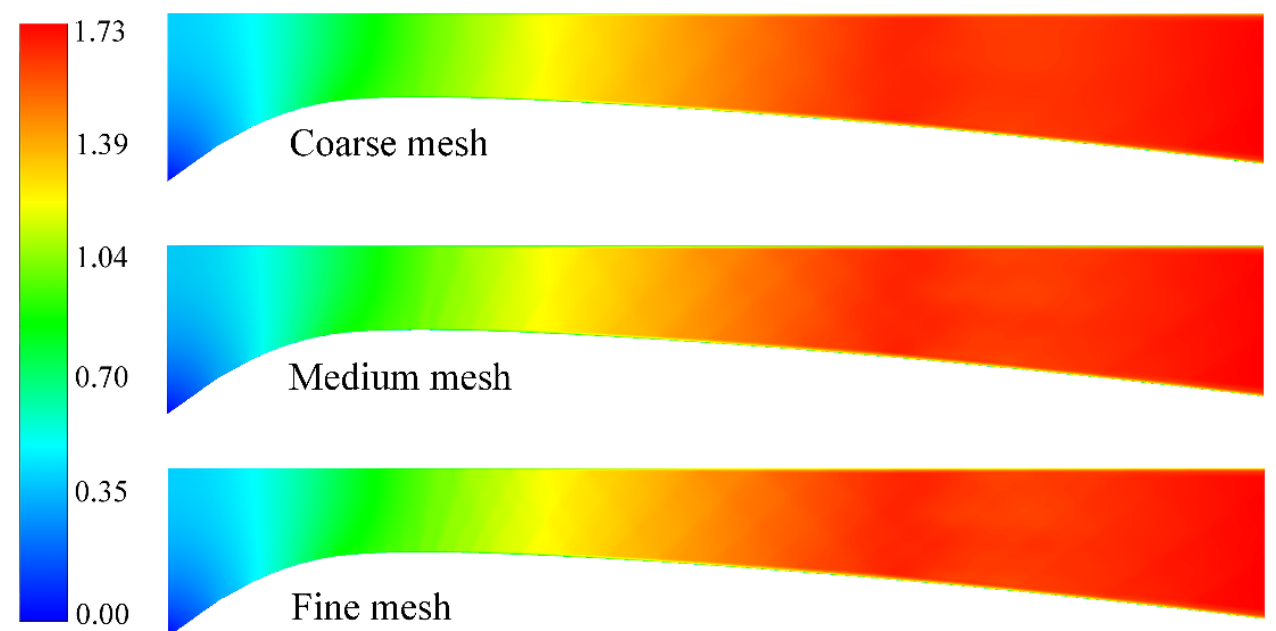

Mach number

(a)

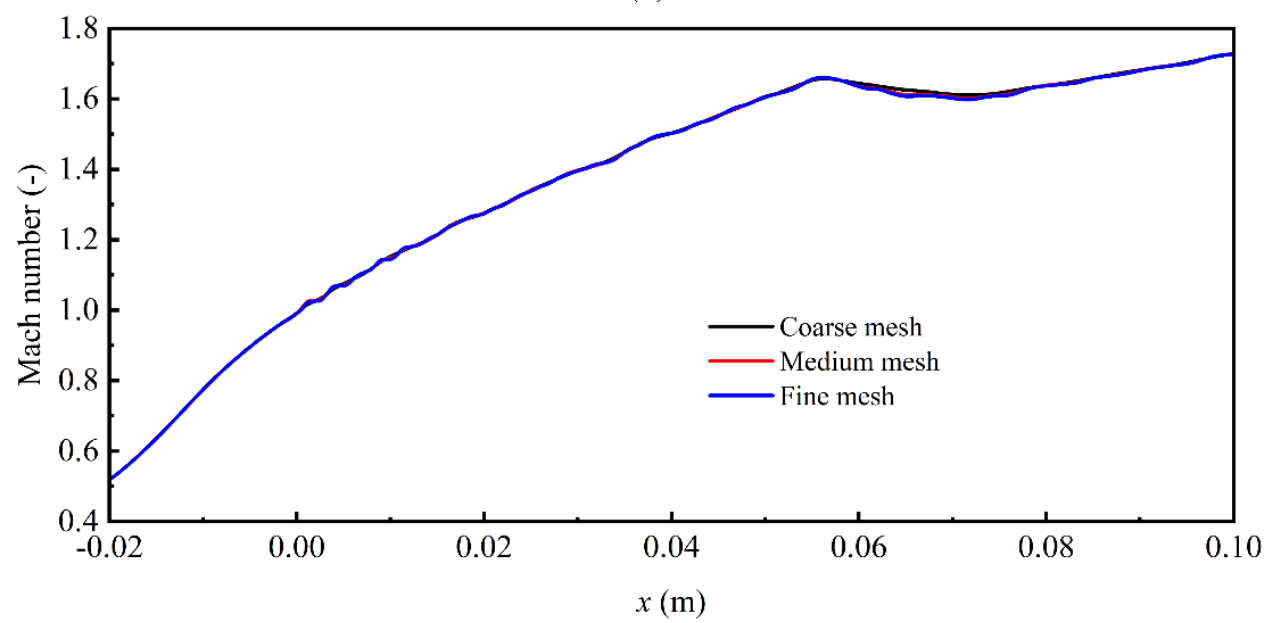

(b)

247 Fig. 5 Mach number in the Hedbäck's nozzle with coarse, medium and fine meshes: 

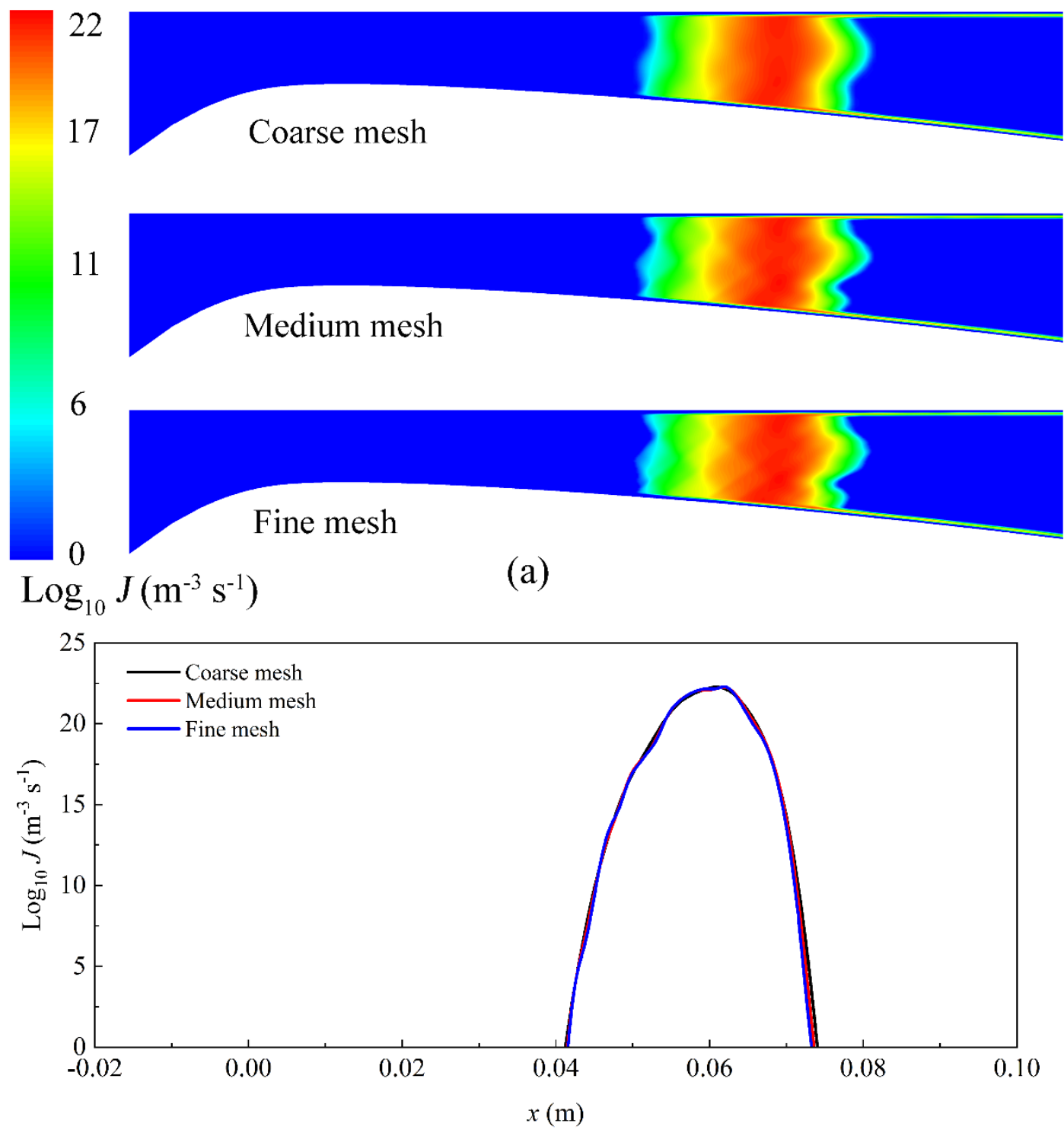

(b)

250 Fig. 6 Nucleation rate in the Hedbäck's nozzle with coarse, medium and fine meshes: 




(a)

19

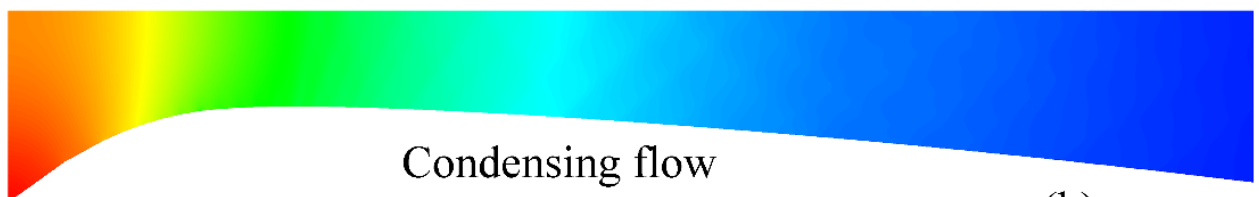

Condensing flow

(b)

Static pressure (bar)

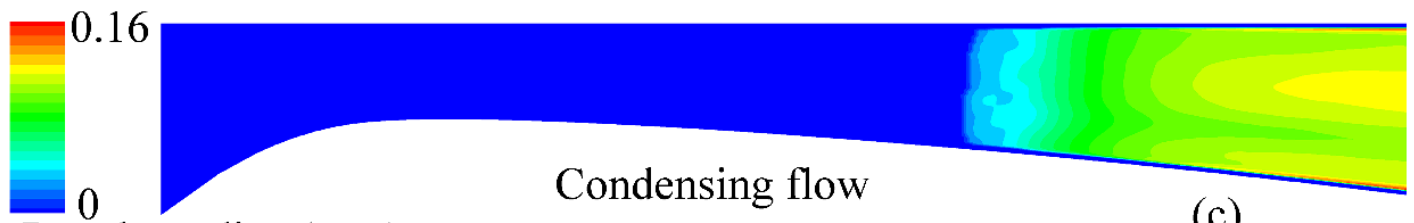

Droplet radius $(\mu \mathrm{m})$

(c)

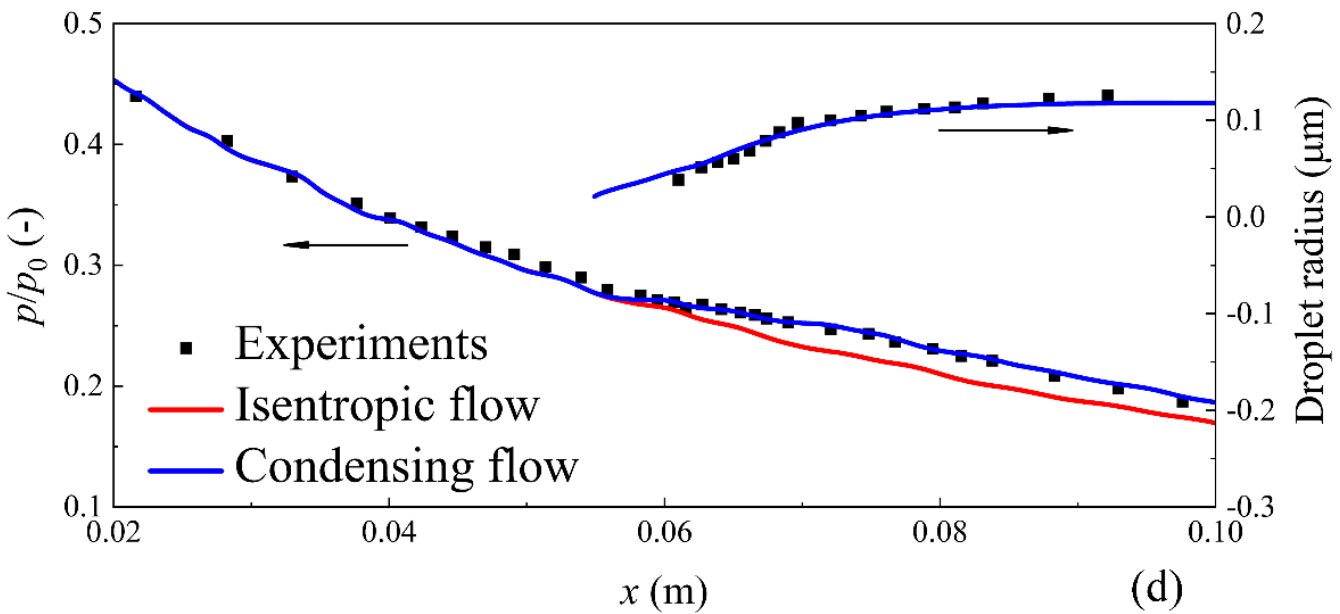

Fig. 7 Numerical and experimental data in the Hedbäck's nozzle: contours of static

254 pressure for the single-phase flow (a), contours of static pressure for condensing flows

255 (b), contours of droplet radius for condensing flows (c), and profiles for the singlephase flow and condensing flows (d)

\section{Results and discussion}

\subsection{Flow structure in supersonic separators}

The flow structure and condensation phenomenon in the supersonic separator will 
8 presents the static pressure and Mach numbers inside the supersonic separator considering non-equilibrium condensation, and the distributions of the condensation parameters are shown in Fig. 9 including the degree of supersaturation, nucleation rate, liquid mass generation rate and liquid fraction. The degree of supersaturation, $S$, describes the saturation level of a vapour, which is defined as the ratio of vapour pressure, $p_{v}$ to the equilibrium saturation pressure at the local temperature, $p_{s}(T)$.

$$
S=\frac{p_{v}}{p_{s}(T)}
$$

It is observed that the gas accelerates in the converging part of the nozzle, which induces the decrease of the static pressure. After the critical condition is achieved at the nozzle throat with a Mach number of unity, the fluid is further expanded to the supersonic flow in the diverging part of the nozzle. During this process, the degree of supersaturation increases slowly to the saturation state where the nucleation does not appear immediately. Then the vapour phase goes into non-equilibrium state and the degree of supersaturation rises sharply to a maximum value of 4.28. Non-equilibrium state of the vapour induces the occurrence of the vapour nucleation, which starts to condense at a degree of supersaturation of 2.54. The sharp profile of the nucleation rate means that the condensation is accomplished in an extremely short time with the peak value of $10^{21} \mathrm{~kg} \mathrm{~m}^{-3} \mathrm{~s}^{-1}$ and the vapour phase goes back to an equilibrium state. It can be noticed that the maximum nucleation rate and degree of supersaturation occur at the same axial location of the supersonic separator.

The liquid mass generation rate starts to increase rapidly after the nucleation process is initiated due to the extremely non-equilibrium state. The comparison from 
the profiles of the nucleation rate and liquid mass generation rate indicates that the generation of the liquid phase occurs downstream of the location where the nucleation process starts. The liquid fraction smoothly increases to the maximum value of about 0.034 as a result of the existence of the positive liquid mass generation rate until the appearance of the shock wave in the diffuser, which results in the increase of the static pressure and temperature, and the subsonic flow is achieved under this condition. The liquid fraction decreases to zero value, which signifies that the condensed liquid goes back to the gaseous state.

In the numerical simulation of the wet steam in supersonic flows without considering a shock wave, the degree of subcooling remains around $5 \mathrm{~K}$ after the intense nucleation process $[38,39]$. This indicates that the vapour molecules continue to aggregate on the surface of the nuclei, which results in the growth of the condensed droplet [40]. Correspondingly, the liquid fraction increases in the diverging part of the supersonic nozzle. On the contrary, the appearance of the shock wave produces the rapid increase of the static pressure and temperature, which leads to the equilibrium state of the vapour. The re-evaporation of the condensed droplet occurs downstream the shock wave. This will decrease the dehydration performance for the supersonic separator, and therefore, should be controlled during the operation. 




301

302

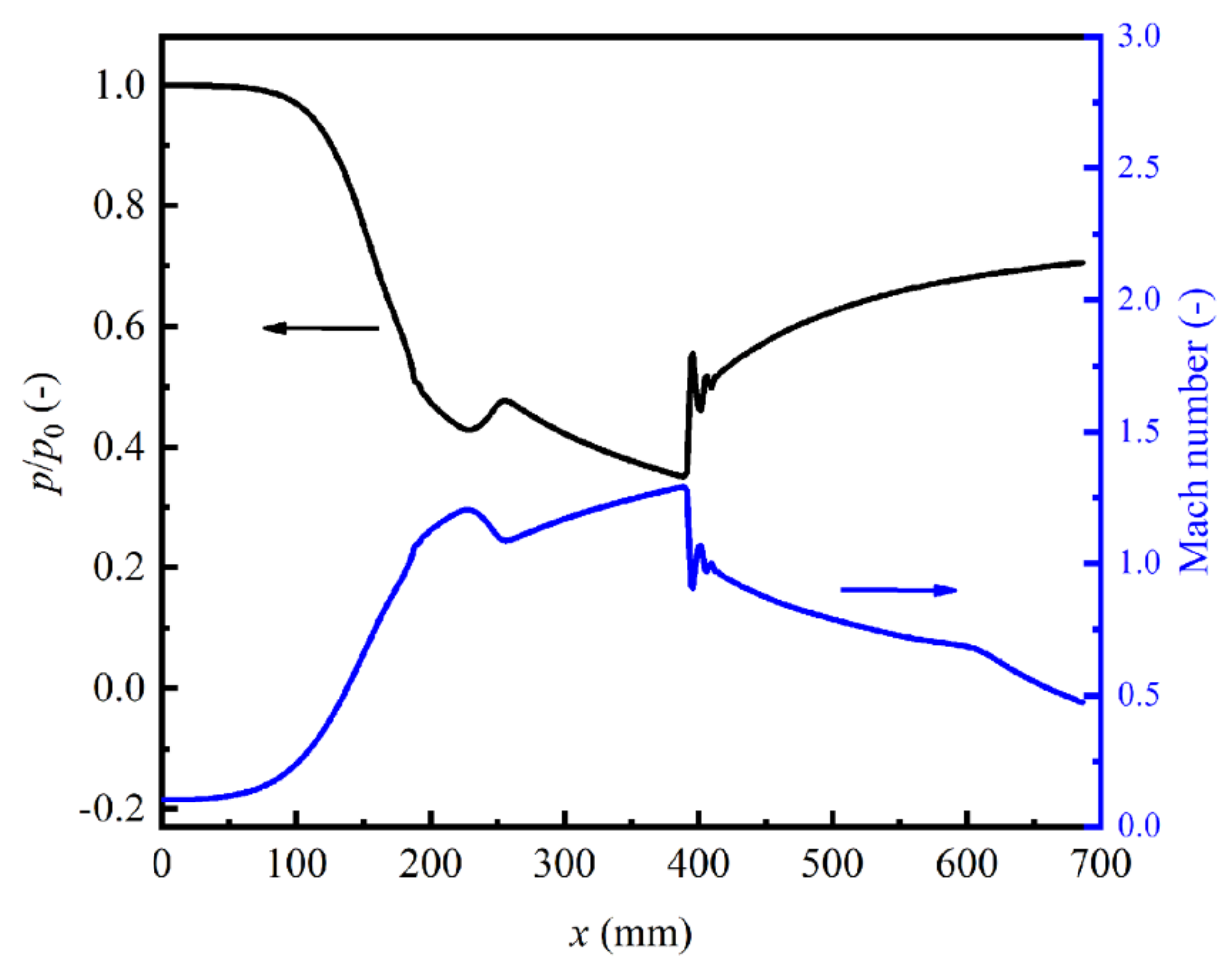

303

304

305

306

(a)

a)

(b)

Fig. 8 Static pressure and Mach number in the supersonic separator: contours (a) and profiles (b)

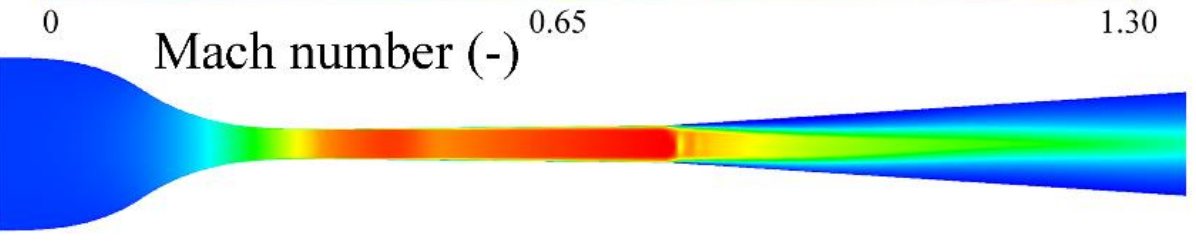






307

(a)



Fig. 9 Condensation parameters in the supersonic separator: nucleation rate and 


\subsection{Effect of inlet subcooling on condensation and shock waves}

The distributions of subcooling, static pressure and entropy at the central line of the supersonic separator are described in Fig. 10 under the condition of different inlet subcooling values. One can notice that higher subcooling values result in a higher degree of supersaturation at the entrance of the supersonic separator. The vapour with a less inlet subcooling correspondingly needs to travel further into the nozzle to start non-equilibrium condensation in supersonic flows. The increasing inlet subcooling shifts the onset of non-equilibrium condensation towards the nozzle throat. Combining the static pressure and the entropy results, it can be observed that the vapour condensation occurs immediately in the highest inlet subcooling after it reaches the critical state at the nozzle throat. Furthermore, the static pressure increases significantly at a higher inlet subcooling value. This indicates that more heat and mass transfer occur during non-equilibrium condensation process, which is demonstrated by the distribution of the entropy at various inlet subcooling values.

The condensation parameters are described in Fig. 11 at various inlet subcooling values including the nucleation rate, liquid mass generation rate and liquid fraction. For lower inlet subcooling values, the vapour further expands after achieving the critical flow at the nozzle throat without experiencing an immediate condensation. The lower static temperature is obtained as the vapour goes further in the divergent part of the Laval nozzle before the appearance of the nucleation. This consequently results in a higher degree of supersaturation for non-equilibrium condensation, which induces a 
later onset of the nucleation rate. Based on the aforementioned analysis, it can be concluded that the vapour with higher inlet subcooling starts the condensation at a lower degree of supersaturation. The generation rate of liquid mass decreases with the lower inlet subcooling during the processes of nucleation and condensation. After reaching the peak, the liquid mass generation rate decreases dramatically due to the release of the latent heat. More liquids are condensed at a higher inlet subcooling value during non-equilibrium condensation processes.

Furthermore, the shock wave position varies significantly under different inlet subcooling values as shown in Fig. 10 and Fig. 11. The decrease of the inlet subcooling shifts upstream the shock wave position. For instance, the shock wave occurs at $x=382$ $\mathrm{mm}$ for the inlet subcooling of $-11 \mathrm{~K}$ while it moves upstream to $x=371 \mathrm{~mm}$ with the inlet subcooling decreasing to $-26 \mathrm{~K}$. This upstream movement will result in the occurrence of the shock wave in the diverging part of the supersonic nozzle, in which the condensed liquid will re-evaporate due to the increase of the pressure and temperature downstream the shock wave. Consequently, the water vapour will not be removed by the supersonic separator. This indicates that the dehydration performance of the supersonic separator can be improved by increasing the inlet subcooling in natural gas processing both from the view of liquid generation and shock wave position. 


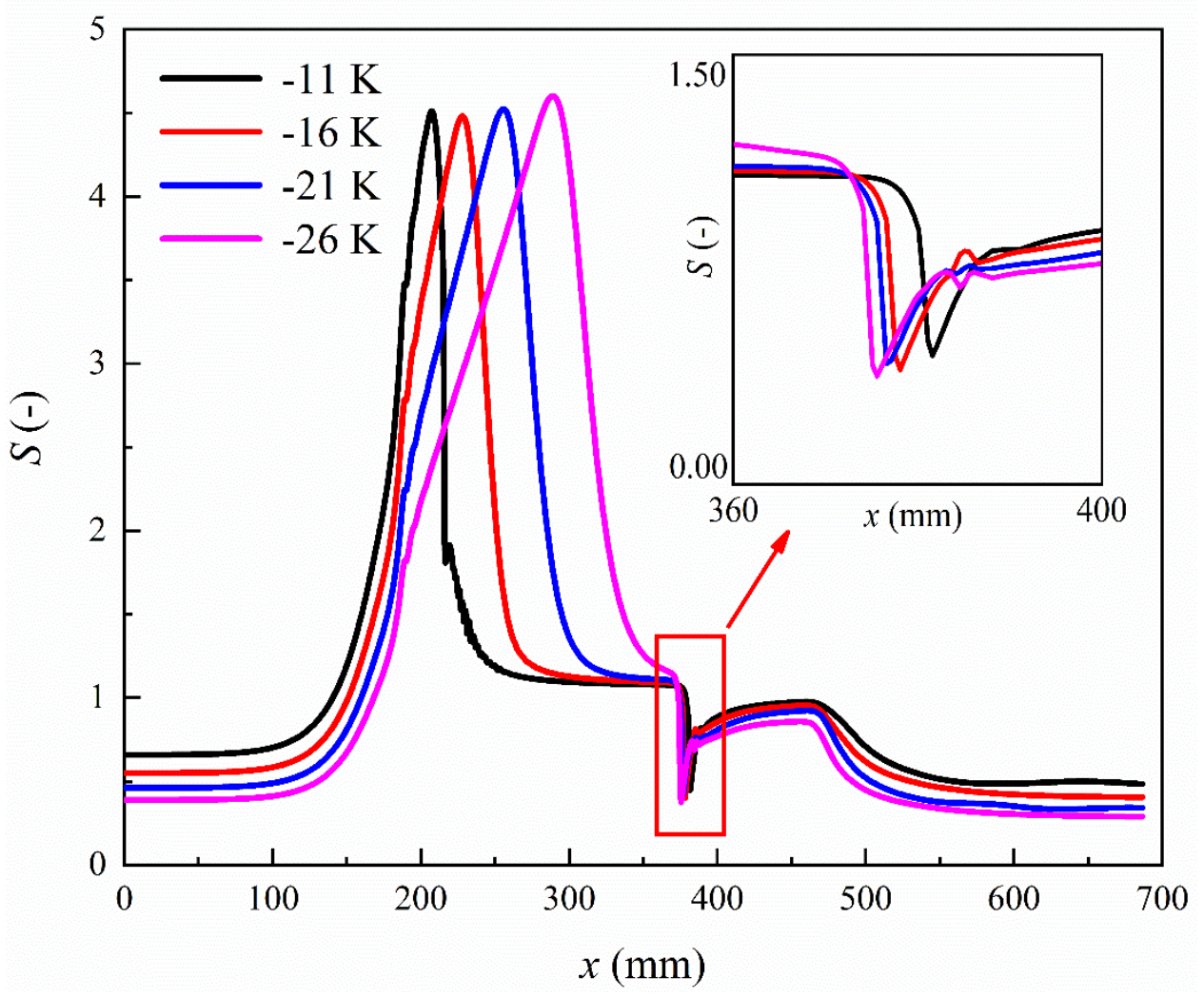

353

(a)

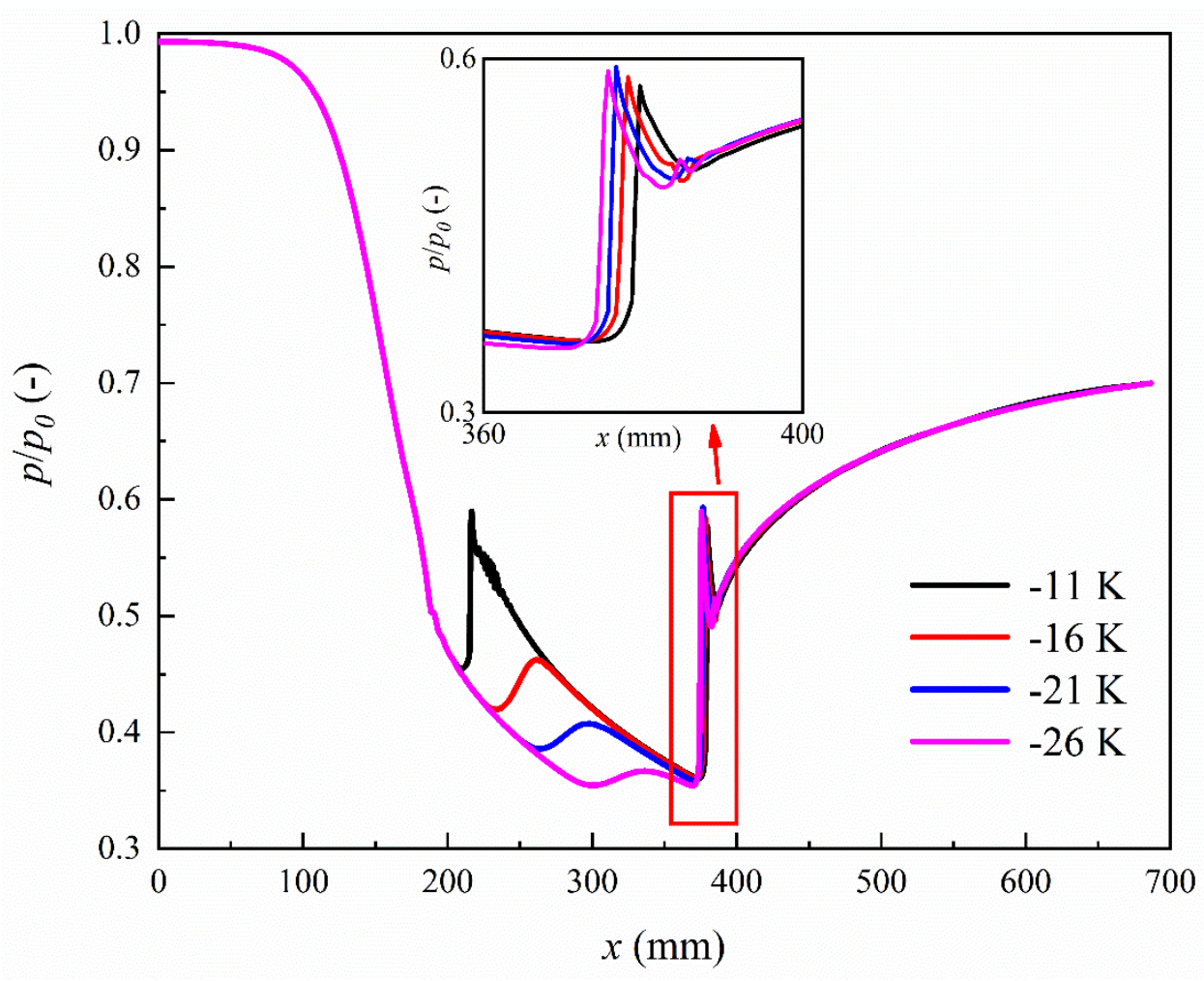




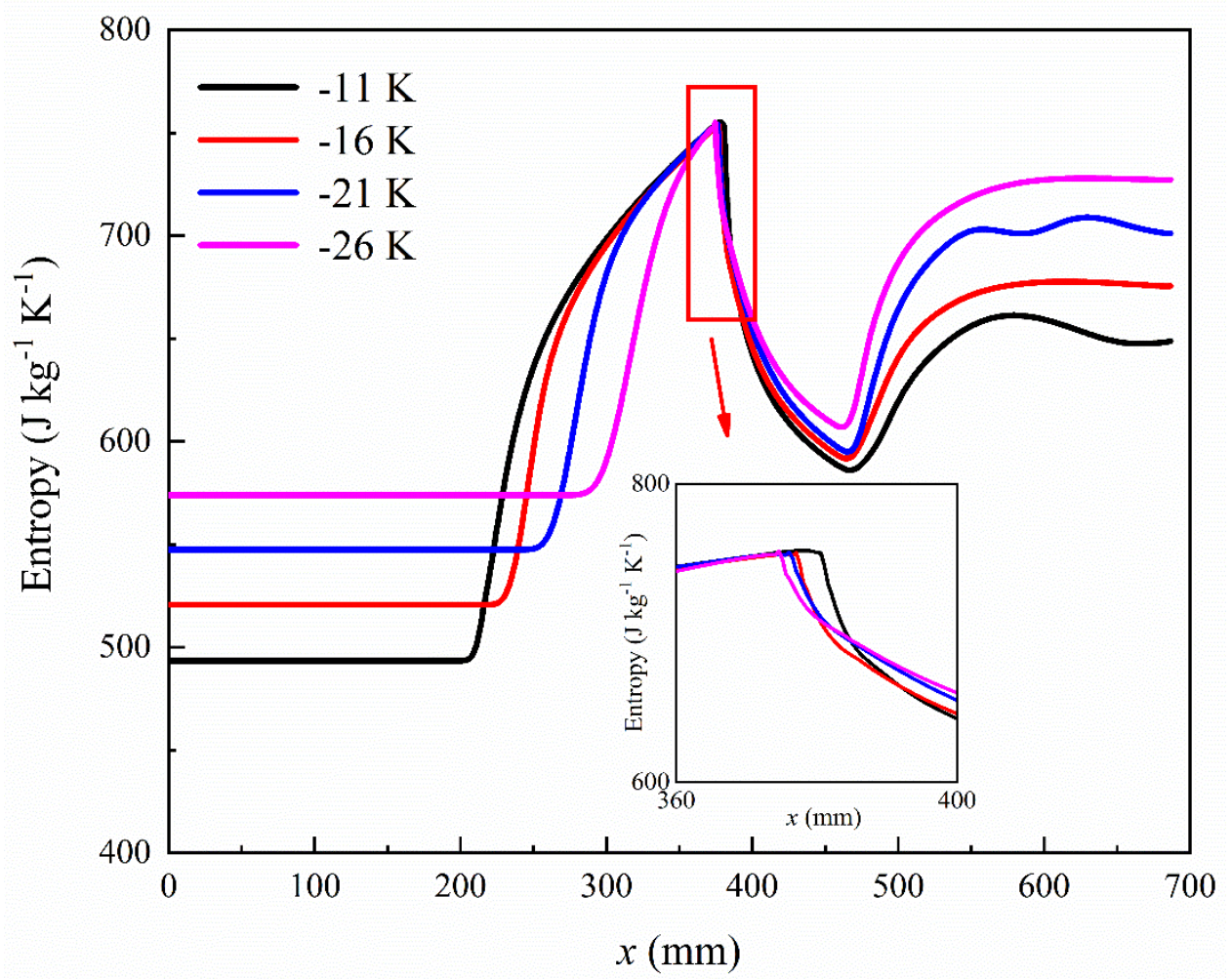

356

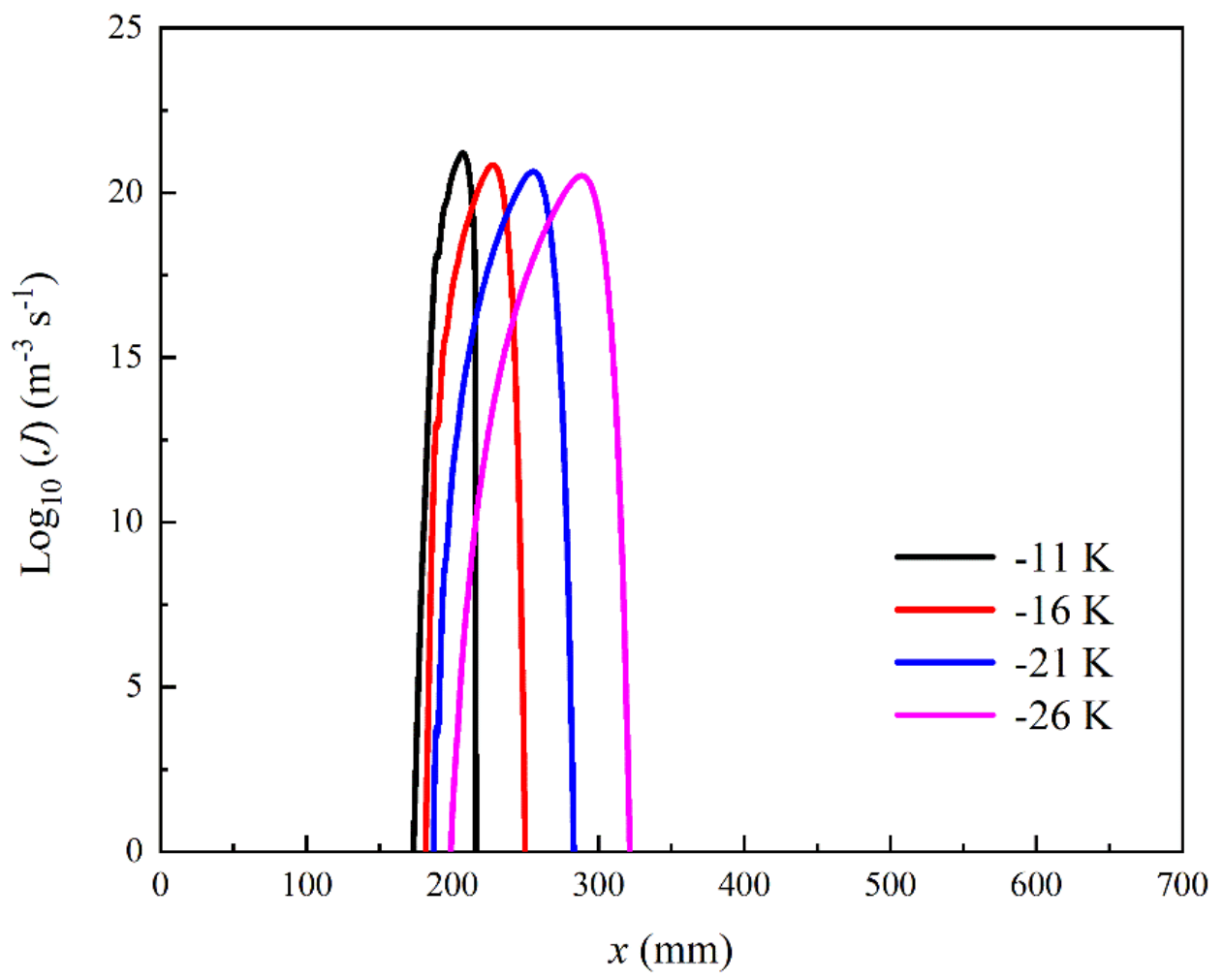

Fig. 10 Degree of supersaturation (a), static pressure (b) and entropy (c) at various inlet subcooling values in the supersonic separator 
(a)

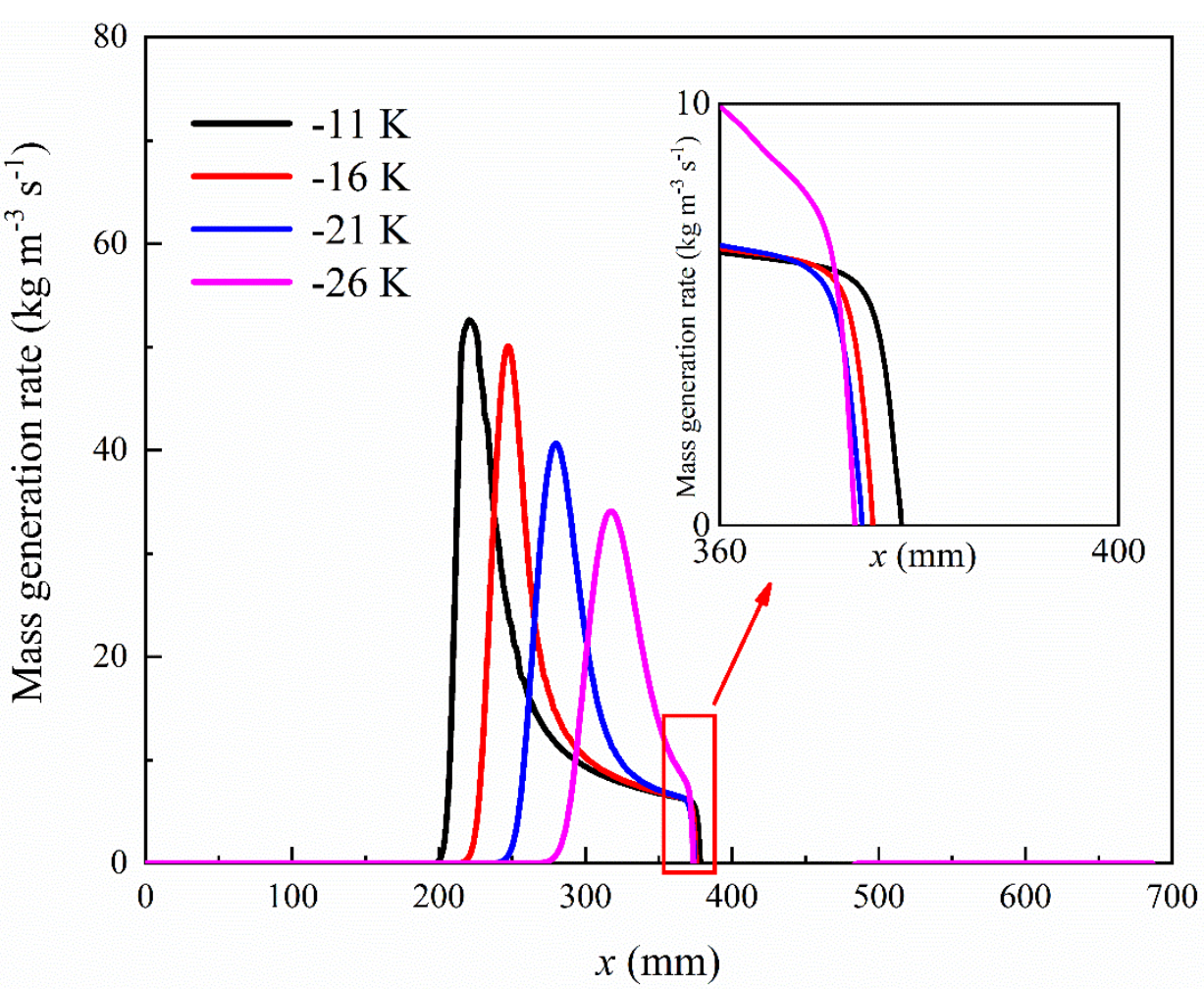

362

(b)

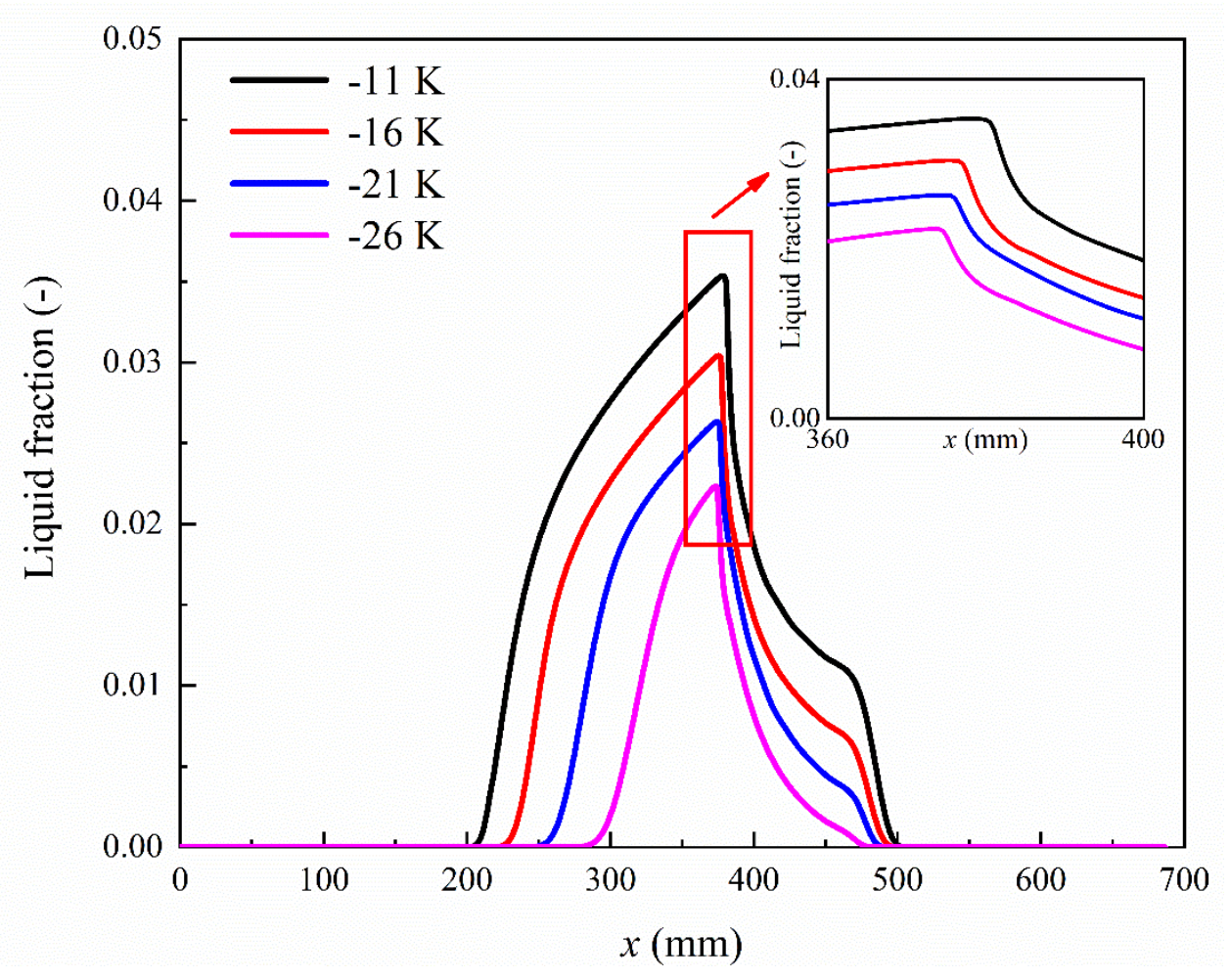


Fig. 11 Nucleation rate (a), mass generation rate (b) and liquid fraction (c) at various inlet subcooling values in the supersonic separator

\subsection{Effect of inlet saturation on condensation and shock waves}

Figure 12 presents the effect of the inlet saturation on the condensation parameter of water vapour inside the supersonic separator, including the nucleation rate, liquid mass generation rate, droplet radius and liquid fraction, degree of supersaturation and static pressure. The numerical study shows that the higher inlet saturation induces an earlier onset of non-equilibrium condensation. The nucleation starts near the downstream of the nozzle throat at the highest inlet saturation of 0.55 . It is also observed that the higher liquid mass generation rate and droplet radius are obtained with the increase of the inlet saturation value, which leads to an increase of the liquid fraction. For example, the liquid fraction is approximately 0.021 of the total mass with the inlet saturation of 0.40 . The liquid fraction increases by approximately $50 \%$ to 0.031 of the total mass, when the inlet saturation is increased to 0.55 .

The inlet saturation also influences remarkably the position of the shock wave in the supersonic separator. The shock wave moves downstream from $x=366 \mathrm{~mm}$ to $x=$ $378 \mathrm{~mm}$ with the increase of the inlet saturation from $S=0.40$ to $S=0.55$. This indicates that the supersonic separator can benefit from the high inlet pressure of the vapour during the natural gas dehydration processes. Based on the numerical evaluation of the inlet saturation, it is suggested that the supersonic separator can replace the JouleThomson valve, which is usually installed in the wellhead to decrease the high pressure of the natural gas. Therefore, the supersonic separator can maximise the utilisation of 
the pressure energy to achieve the goal of energy conservation for gas processing.



(a)






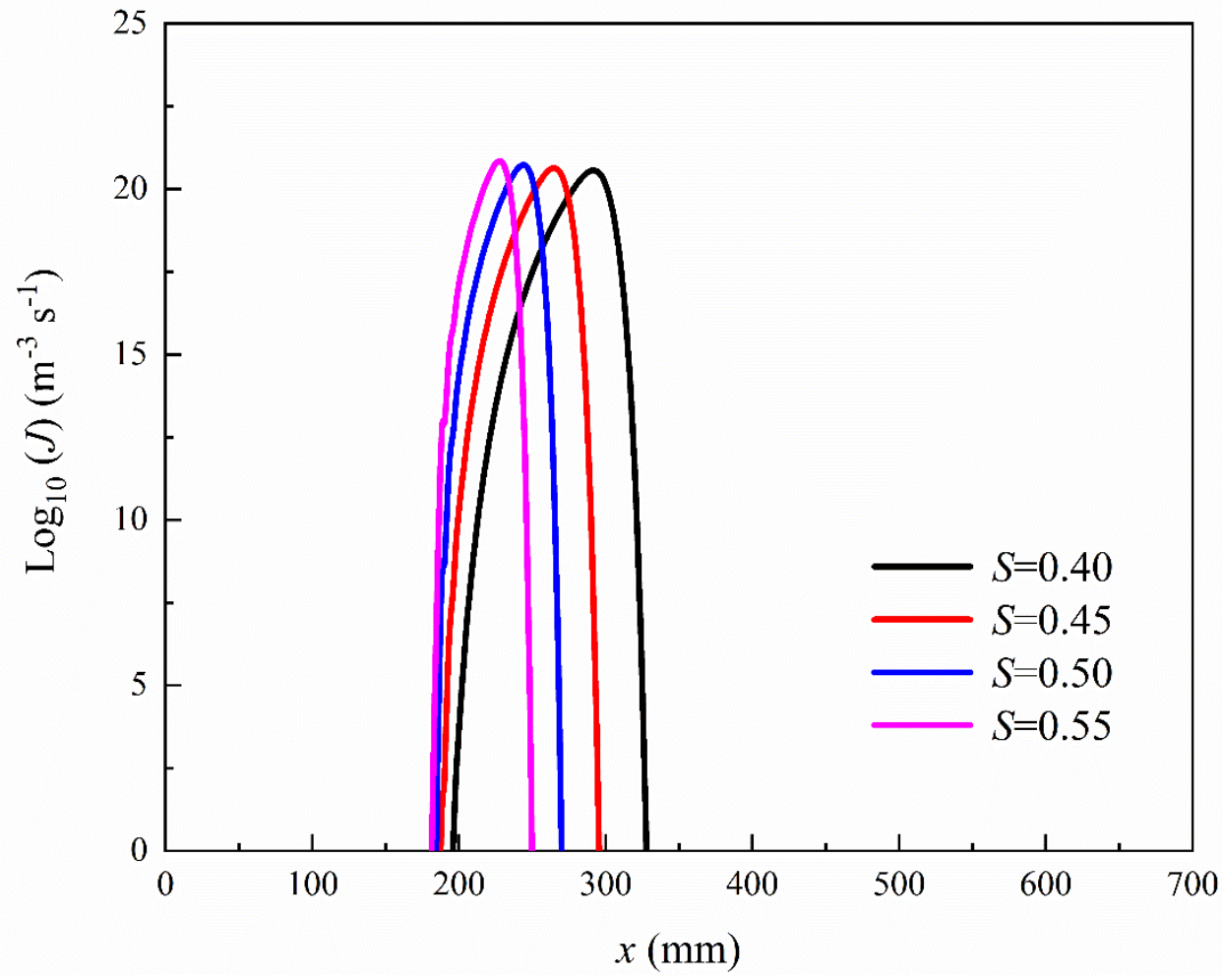

393 394

(c)



395

(d) 


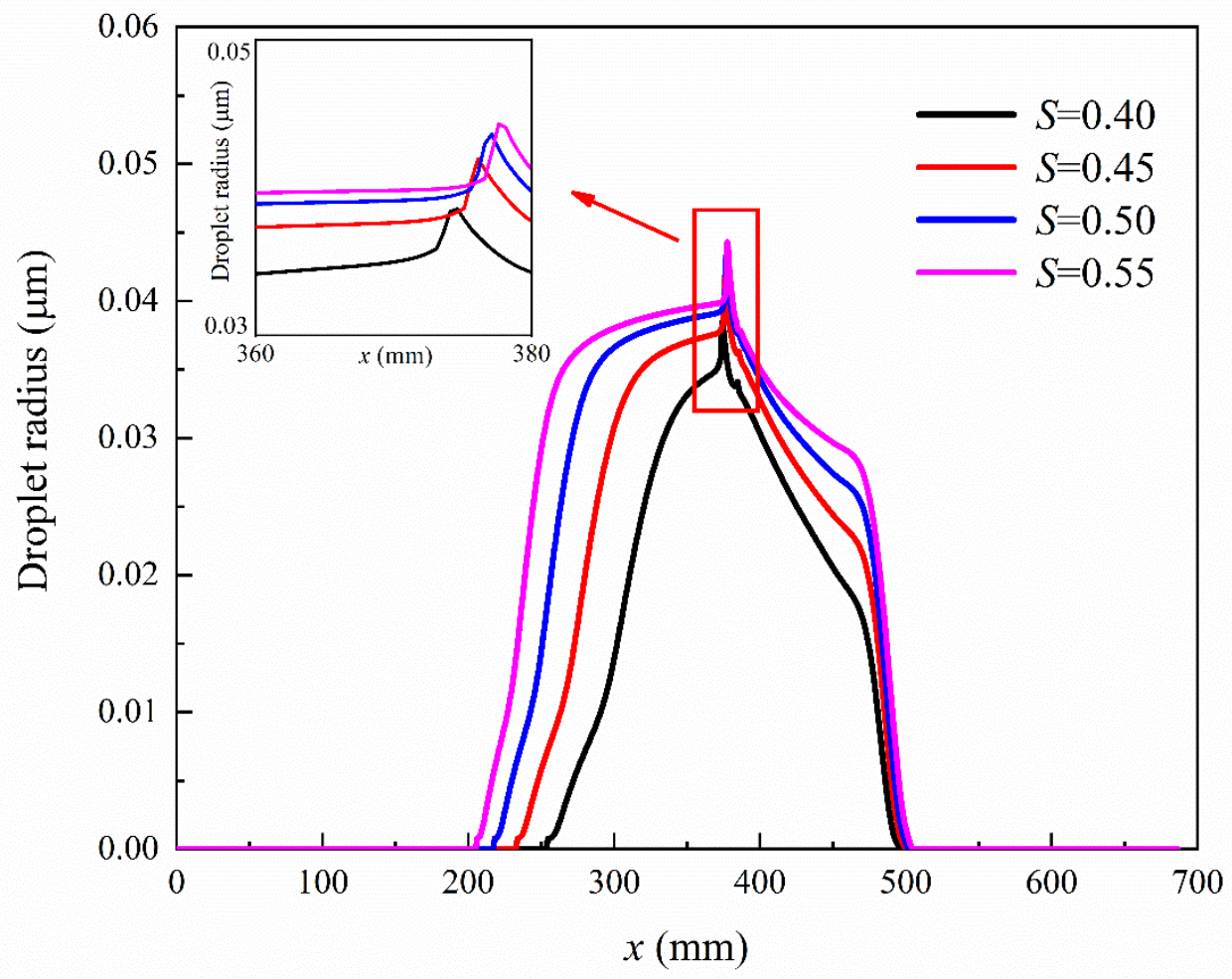

397

(e)

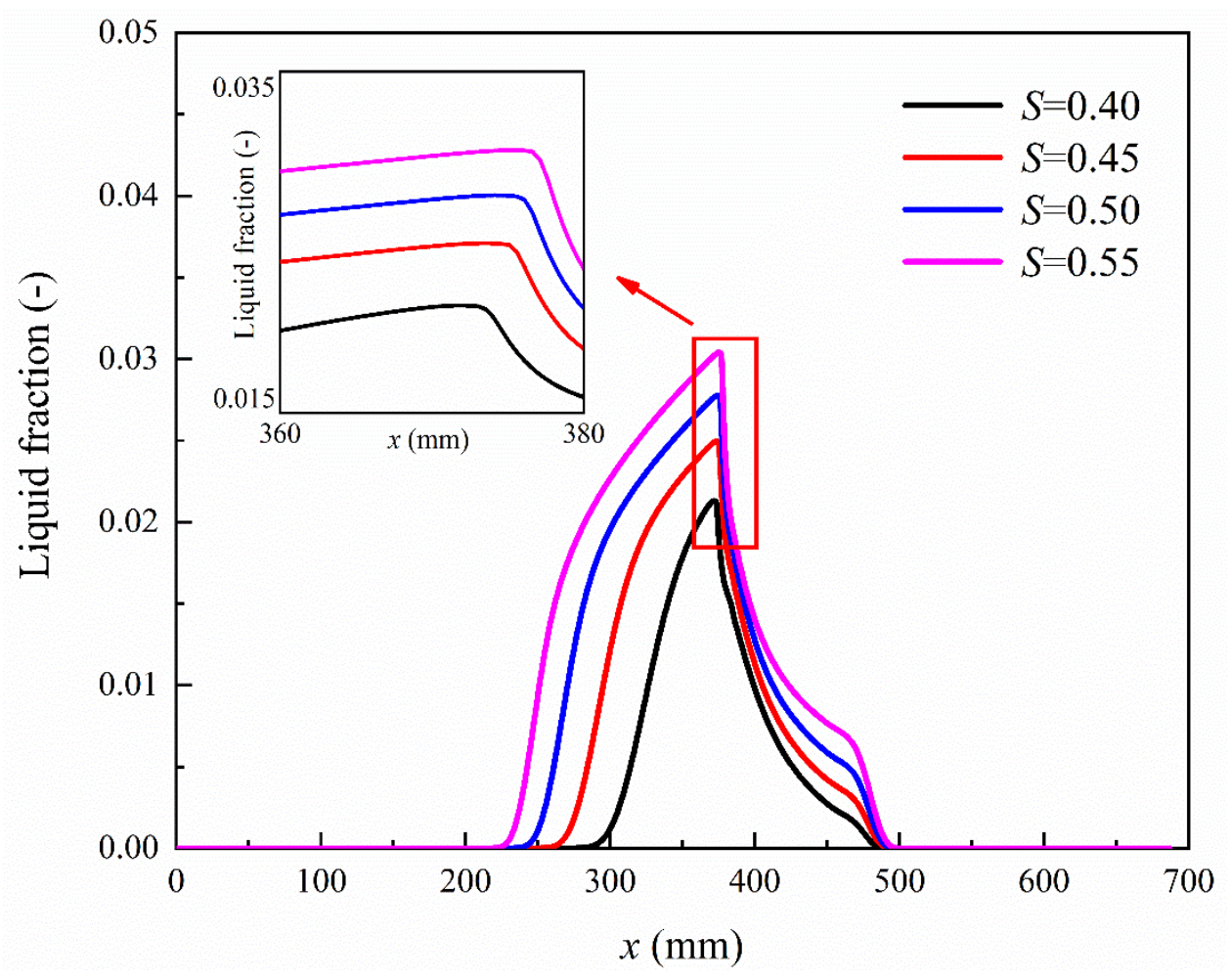

399

(f)

Fig. 12 Condensation parameters in the supersonic separator at various inlet 
supersaturations: degree of supersaturation (a), static pressure(b), nucleation rate (c), mass generation rate (d), droplet radius (e) and liquid fraction (f)

\subsection{Effect of shock waves on condensation processes}

In this section, the effect of the shock wave on the non-equilibrium condensation is investigated in supersonic flows at low inlet subcooling of $-34 \mathrm{~K}$ and low inlet saturation of 0.28 . Figure 13 illustrates the contours and profiles of Mach number, degree of supersaturation and nucleation rate, respectively. The computational result shows that the low inlet subcooling and inlet saturation shift non-equilibrium condensation to the entrance of the diffuser, where a shock wave occurs. It is observed that a shock wave intersects the region of the intense nucleation process. In the presence of a shock wave, subsonic flows are generated downstream the shock wave, which induces an increase of the static pressure and static temperature. Accordingly, the degree of supersaturation represents a fluctuation due to the strong shock wave and the value is less than unity further downstream the shock wave. This indicates that the vapour-liquid two-phase flow returns towards thermodynamic equilibrium. Furthermore, the distribution of the nucleation rate is sharply decreased due to the occurrence of the shock wave, which is similar to the distributions of Mach number and degree of supersaturation. This indicates that the nucleation process is terminated and non-equilibrium condensation process is not completed. Compared to the distribution of the nucleation rate in Fig. 9 (a), in which the condensation process occurs naturally, it demonstrates that the nucleation process ceases at the cut-off point. Under this condition, the condensed droplets re-evaporate and the water vapour can not be 
424 removed from the natural gas, which should be prevented in the normal operation of 425 the supersonic separator.



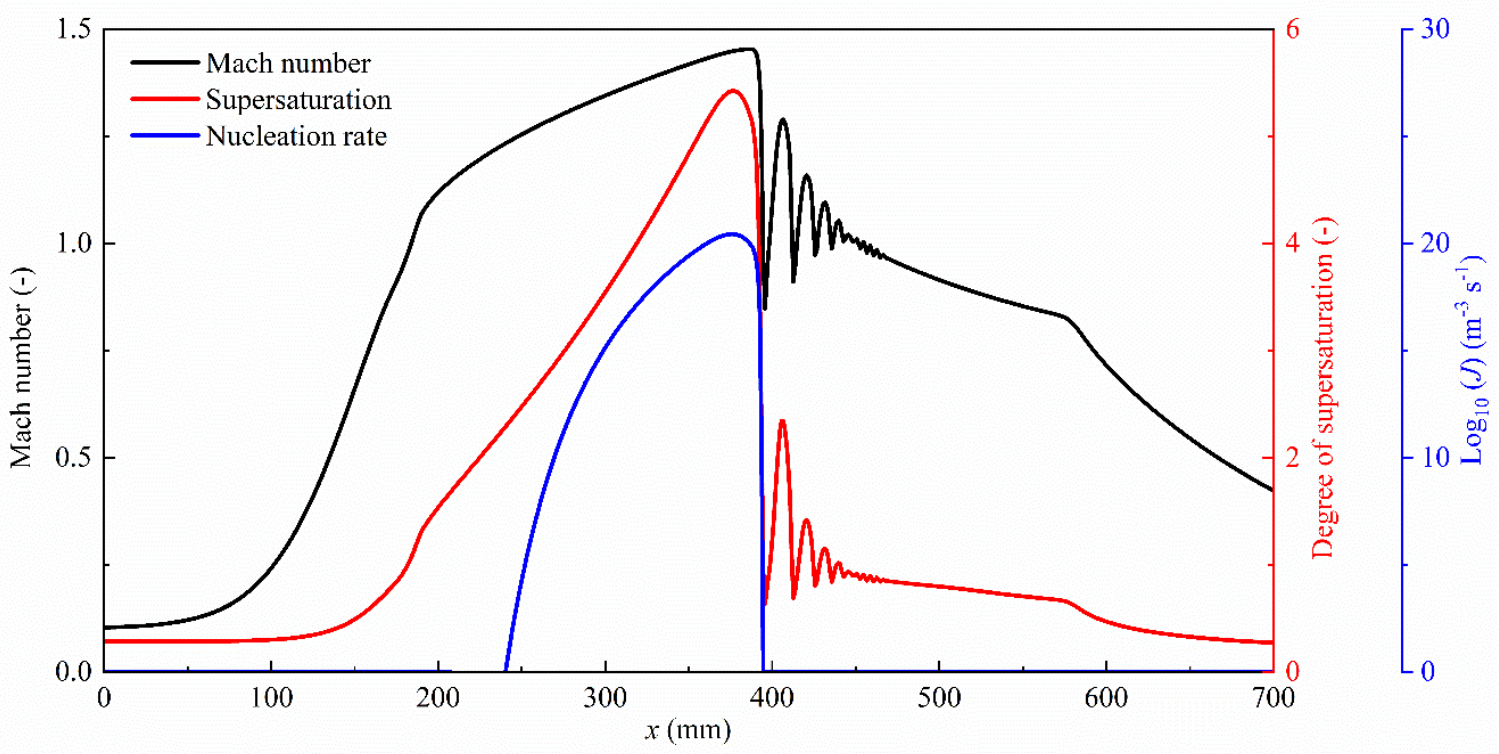

(b)

Fig. 13 Contours (a) and profiles (b) of Mach number, degree of supersaturation and nucleation rate during the interaction between condensation process and shock waves in supersonic flows

\subsection{Effect of swirling flows on the condensation process}

The swirling flow is one of the key features for the supersonic separator, which generates a strong centrifugal force to remove the condensed liquid from gas mixtures. In the ANSYS FLUENT [32], the axisymmetric swirl model is used to simulate the pseudo-3D model by implementing a tangential component of the gas velocity at the inlet boundary. Thus, the axisymmetric swirl model is employed to evaluate the effect of the swirling flow on the non-equilibrium condensation process in the supersonic separator, which means that we do not need to install static vanes to generate the swirling flows. The swirl number, $S_{w}$, which can be used to quantify the strength of a swirling flow, is defined as the ratio of the maximum tangential velocity, $w m$, to the sound speed at the nozzle throat, $a_{t h}$. 


$$
S_{w}=\frac{w_{m}}{a_{t h}}
$$

Figure 14 illustrates the contours and profiles of the swirl velocity inside the supersonic separator based on three different strengthes of the swirling flow of no swirl $\left(S_{w}=0\right)$, medium swirl $\left(S_{w}=0.15\right)$ and high swirl $\left(S_{w}=0.34\right)$. The case of "no swirl" is a basic simulation without a tangential component of gas velocity at the inlet of the supersonic separator. For both the medium swirl and high swirl, the maximum swirl velocity is obtained at the nozzle throat. This is because the swirl velocity increases due to the converging profile of the supersonic nozzle, while the diverging shape of the supersonic nozzle weakens the swirling flow. The maximum swirl velocities for medium swirl and high swirl are approximately $66 \mathrm{~m} / \mathrm{s}$ and $150 \mathrm{~m} / \mathrm{s}$, respectively. The appearance of the swirling flow induces a nonuniform distribution of the fluid flow, which can be demonstrated by the contours and profiles of the static pressure in Fig. 15. It can be observed that the static pressure is uniformly distributed at the cross-section of the supersonic separator. The stronger swirling flow represents a higher gradient of the static pressure at the cross-section.

The swirling flow also influences the non-equilibrium condensation process of the water vapour in supersonic flows. Figure 16 and Figure 17 describe the distributions of the nucleation rate and liquid fraction under three different strengthes of the swirling flow, respectively. We can see that the increasing swirling flow decreases the magnitude of the nucleation rate. For instance, the maximum nucleation rate can reach $1.2 \times 10^{22}$ $\mathrm{m}^{-3} \mathrm{~s}^{-1}$ without a swirling flow, while it is only about $1.9 \times 10^{21} \mathrm{~m}^{-3} \mathrm{~s}^{-1}$ for the "high swirl" case. Correspondingly, this flow behaviour influences the distribution of the 
Swirl velocity $(\mathrm{m} / \mathrm{s})$



(b)

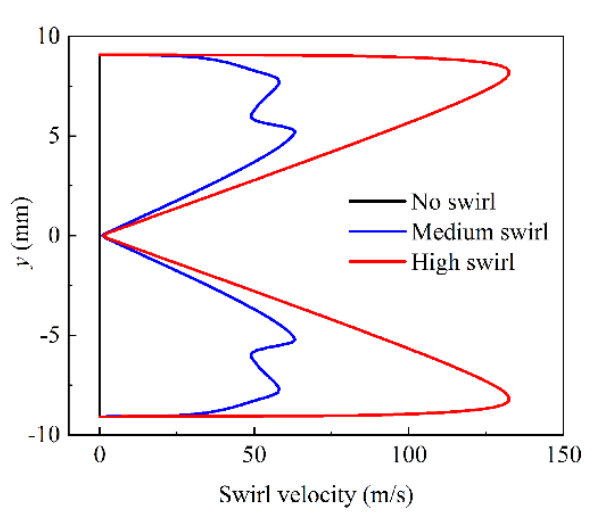

(c)

Fig. 14 Swirl velocity in the supersonic separator: contours (a), profiles along the flow direction at the radial direction $y=5 \mathrm{~mm}$ (b) and profiles at the cross-section of $x=$ 

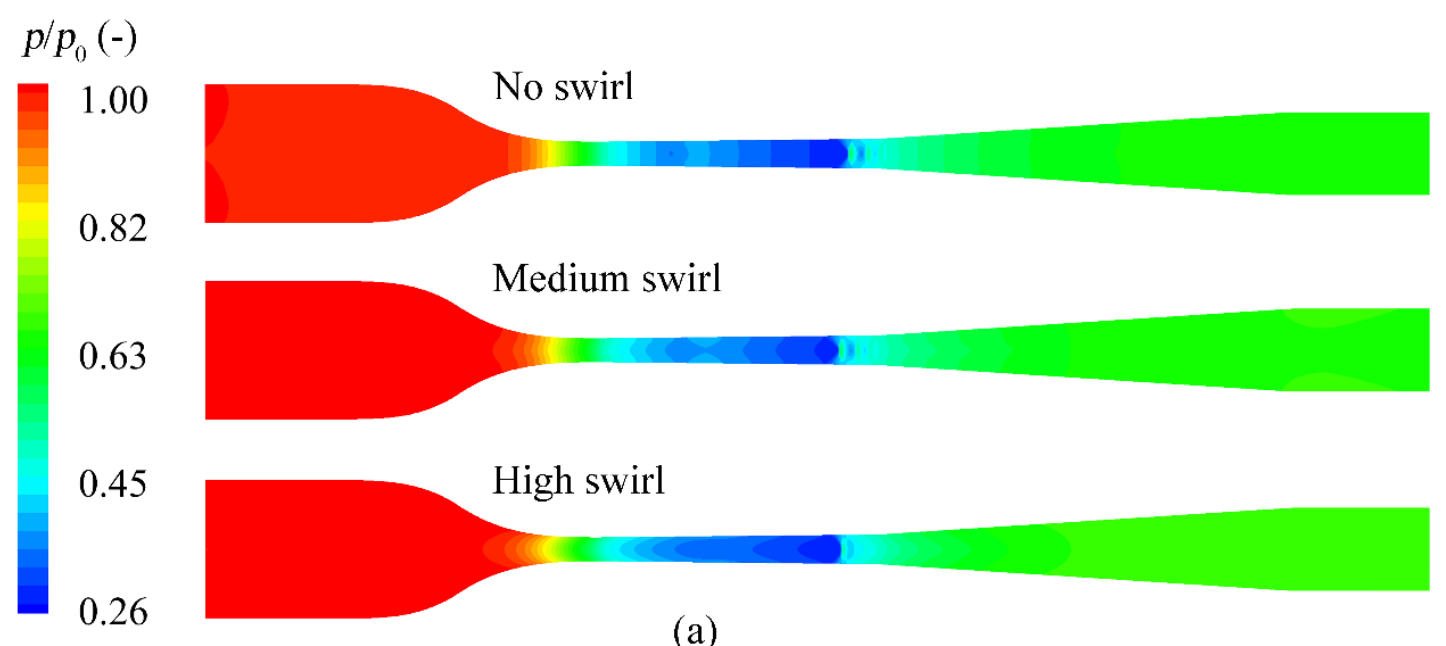

(a)

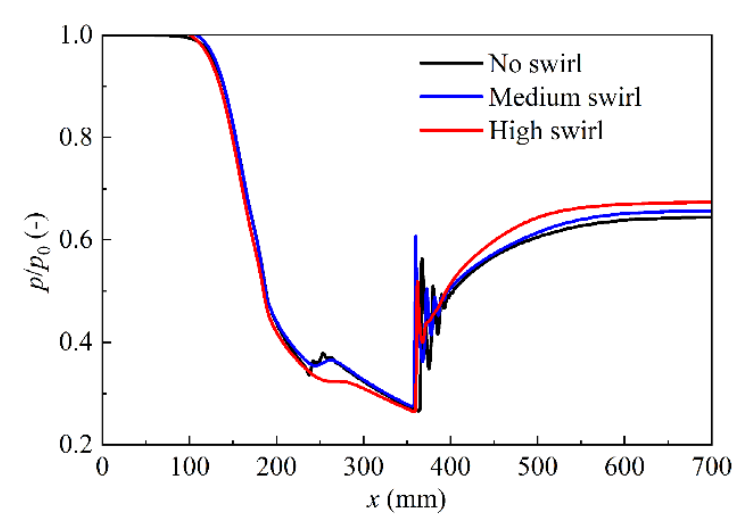

(b)

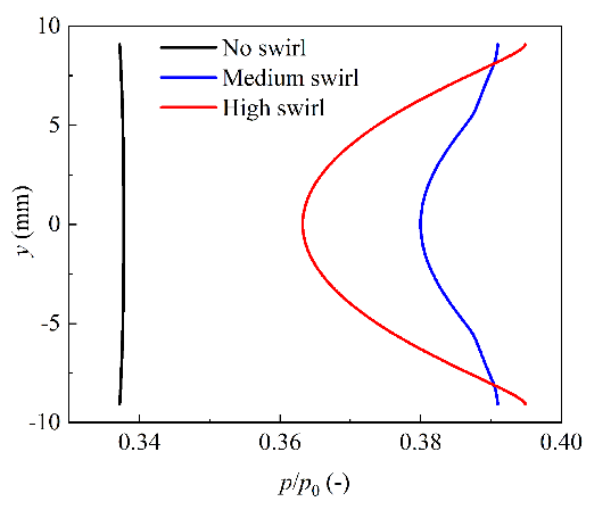

(c)

Fig. 15 Static pressure in the supersonic separator: contours (a), profiles along the

$\log _{10}(J)\left(\mathrm{m}^{-3} \mathrm{~s}^{-1}\right)$
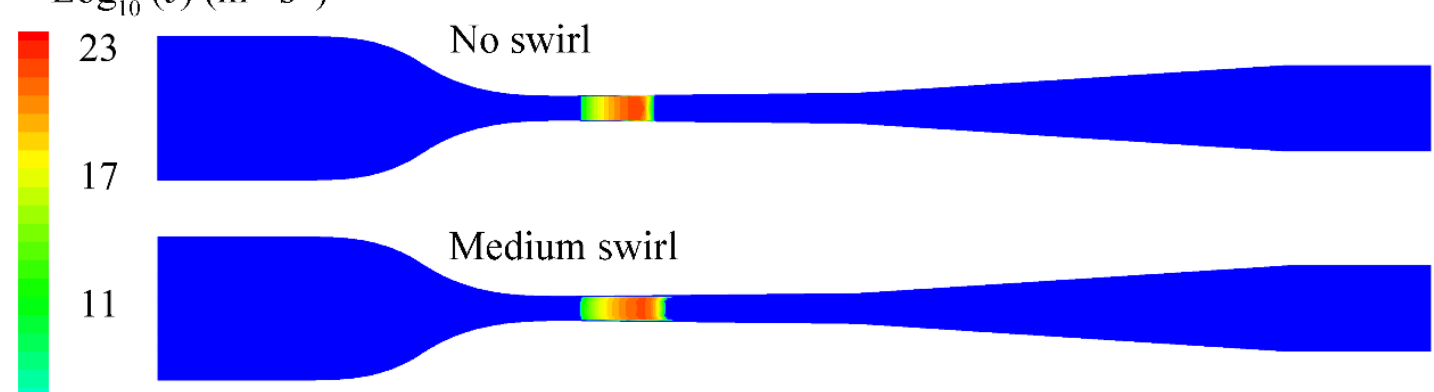

High swirl 


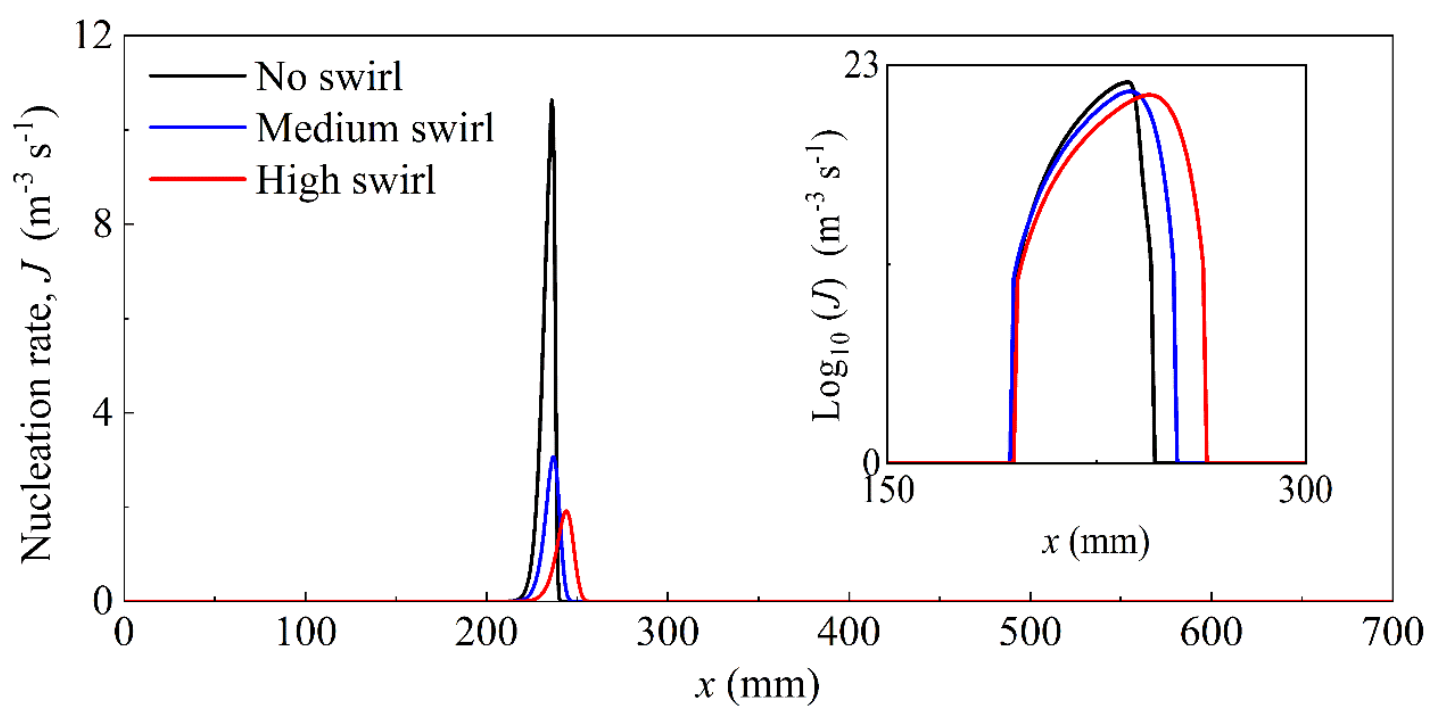

483

(b)

485 Fig. 16 Contours (a) and profiles (b) of the nucleation rate in the supersonic separator 486 under different strengthes of swirling flows

Liquid fraction (-)






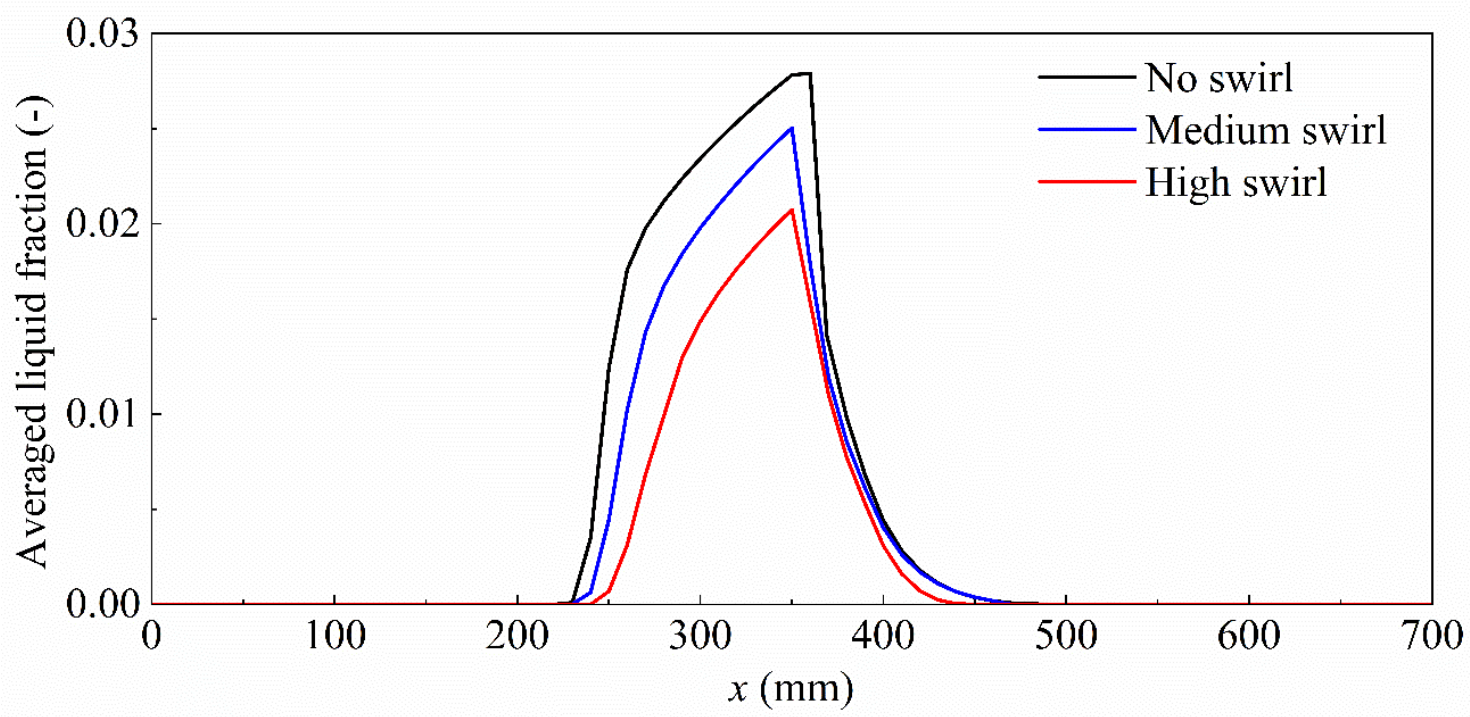

489

490

491

492

493

494

495

496

497

498

499

500

501

502

503

504

(b)

Fig. 17 Contours (a) and profiles (b) of the liquid fraction in the supersonic separator under different strengthes of swirling flows

\section{Conclusions}

The fluid flow in a supersonic separator is evaluated using computational fluid dynamics modelling considering the co-existence of non-equilibrium condensation and shock wave phenomena. In the supersonic separator, water vapour reaches nonequilibrium state, which induces the occurrence of the nucleation process at the degree of supersaturation of 2.54. The maximum degree of supersaturation and nucleation rate are 4.28 and $10^{21} \mathrm{~kg} \mathrm{~m}^{-3} \mathrm{~s}^{-1}$, respectively. The generation of the liquid phase occurs downstream of the position at which the nucleation process takes place. The liquid fraction achieves the maximum value of 0.034 and decreases rapidly to zero value downstream of the shock wave, which means that the occurrence of the re-evaporation of the condensed liquid.

The increasing inlet subcooling and inlet saturation shift downstream the shock 
wave position, and they also induce a higher liquid fraction. The shock wave intersects the region of the intense nucleation process at low inlet subcooling of $-34 \mathrm{~K}$ and inlet saturation of 0.28 . This indicates that the nucleation process is terminated by the shock wave and non-equilibrium condensation process is not completed. This study suggests that the dehydration performance of the supersonic separator can be improved by controlling the inlet pressure and inlet temperature of the gas flow during natural gas processing.

The appearance of the swirling flow leads to the pressure gradient at the crosssection and influence the non-equilibrium condensation process in supersonic separators. The nucleation rate of water vapour decreases with the increase of the swirling flow. Furthermore, the liquid fraction is weakened by $25 \%$ due to the strong swirling flow with a maximum swirl velocity of $150 \mathrm{~m} / \mathrm{s}$ compared to the case without a swirling flow. It suggests that the balance between the swirling flow and condensation process plays a critical role in the removal of water vapour in a supersonic separator.

\section{Acknowledgements}

This project has received funding from the European Union's Horizon 2020 research and innovation programme under the Marie Sklodowska-Curie grant agreement No 792876, the Independent Research Fund Denmark grant 8022-00143B, the Innovation Fund of Denmark and MAN Energy Solutions through the SULCOR project and the National Natural Science Foundation of China under Grant 51876143.

\section{References}

[1] J. Bian, X. Cao, W. Yang, D. Guo, C. Xiang, Prediction of supersonic condensation 
process of methane gas considering real gas effects, Applied Thermal Engineering, $164(2020) 114508$.

[2] D.C. de Melo, L.d.O. Arinelli, J.L. de Medeiros, A.M. Teixeira, G.V. Brigagão, F.M.

[3] J. Hao, C.-Y. Wen, J. Wang, Numerical investigation of hypervelocity shockwave/boundary-layer interactions over a double-wedge configuration, International Journal of Heat and Mass Transfer, 138 (2019) 277-292.

[4] W. Alnoush, M. Castier, Shortcut modeling of natural gas supersonic separation, Journal of Natural Gas Science and Engineering, 65 (2019) 284-300.

[5] S. Dykas, W. Wróblewski, Numerical modelling of steam condensing flow in low and high-pressure nozzles, International Journal of Heat and Mass Transfer, 55(21) (2012) 6191-6199.

[6] A. White, M. Hounslow, Modelling droplet size distributions in polydispersed wetsteam flows, International journal of heat and mass transfer, 43(11) (2000) 18731884.

[7] E. Jabir, B. Dmitrii, A. Konstantin, H.D. Kim, Numerical estimation of nonequilibrium condensation of steam in supersonic nozzles, Journal of Mechanical Science and Technology, 32(10) (2018) 4649-4655.

[8] S. Dykas, W. Wróblewski, Single-and two-fluid models for steam condensing flow 
modeling, International Journal of Multiphase Flow, 37(9) (2011) 1245-1253.

550

551

552

553

554

555

556

557

558

559

560

561

562

563

564

565

566

567

568

569

570

[9] S.M.A. Noori Rahim Abadi, R. Kouhikamali, K. Atashkari, Two-fluid model for simulation of supersonic flow of wet steam within high-pressure nozzles, International Journal of Thermal Sciences, 96 (2015) 173-182.

[10] A. Pillai, B.V.S.S.S. Prasad, Effect of wall surface roughness on condensation shock, International Journal of Thermal Sciences, 132 (2018) 435-445.

[11] A. Zhao, S. Guo, X. Qi, S. Gao, J. Sun, Numerical study on the nano-droplets formation process from superheated steam condensation flow effected by nozzle convergent profile, International Communications in Heat and Mass Transfer, 104 (2019) 109-117.

[12] G. Zhang, X. Zhang, F. Wang, D. Wang, Z. Jin, Z. Zhou, Design and optimization of novel dehumidification strategies based on modified nucleation model in threedimensional cascade, Energy, 187 (2019) 115982.

[13] A. Ebrahimi-Fizik, E. Lakzian, A. Hashemian, Entropy generation analysis of wetsteam flow with variation of expansion rate using NURBS-based meshing technique, International Journal of Heat and Mass Transfer, 139 (2019) 399-411.

[14] Y. Patel, G. Patel, T. Turunen-Saaresti, Influence of turbulence modelling on nonequilibrium condensing flows in nozzle and turbine cascade, International Journal of Heat and Mass Transfer, 88 (2015) 165-180.

[15] W. Wróblewski, S. Dykas, A. Gepert, Steam condensing flow modeling in turbine channels, International Journal of Multiphase Flow, 35(6) (2009) 498-506.

[16] M. Vatanmakan, E. Lakzian, M.R. Mahpeykar, Investigating the entropy 
generation in condensing steam flow in turbine blades with volumetric heating, Energy, 147 (2018) 701-714.

573 [17] H. Ding, Y. Li, E. Lakzian, C. Wen, C. Wang, Entropy generation and exergy 574 destruction in condensing steam flow through turbine blade with surface roughness, Energy Conversion and Management, 196 (2019) 1089-1104.

[18] X. Cao, J. Bian, Supersonic separation technology for natural gas processing: A review, Chemical Engineering and Processing-Process Intensification, 136 (2019)

[19] Q.-F. Ma, D.-P. Hu, J.-Z. Jiang, Z.-H. Qiu, A turbulent Eulerian multi-fluid model for homogeneous nucleation of water vapour in transonic flow, International Journal of Computational Fluid Dynamics, 23(3) (2009) 221-231.

[20] Q.-F. Ma, D.-P. Hu, J.-Z. Jiang, Z.-H. Qiu, Numerical study of the spontaneous nucleation of self-rotational moist gas in a converging-diverging nozzle, International Journal of Computational Fluid Dynamics, 24(1-2) (2010) 29-36.

[21] S.H.R. Shooshtari, A. Shahsavand, Predictions of wet natural gases condensation rates via multi-component and multi-phase simulation of supersonic separators, Korean Journal of Chemical Engineering, 31(10) (2014) 1845-1858.

[22] S.H. Rajaee Shooshtari, A. Shahsavand, Reliable prediction of condensation rates for purification of natural gas via supersonic separators, Separation and Purification Technology, 116 (2013) 458-470.

[23] S.R. Shooshtari, A. Shahsavand, Optimal operation of refrigeration oriented supersonic separators for natural gas dehydration via heterogeneous condensation, 
594 [24] J. Bian, X. Cao, W. Yang, X. Song, C. Xiang, S. Gao, Condensation characteristics 595 of natural gas in the supersonic liquefaction process, Energy, 168 (2019) 99-110.

596 [25] S. Dykas, M. Majkut, K. Smołka, M. Strozik, Study of the wet steam flow in the 597 blade tip rotor linear blade cascade, International Journal of Heat and Mass Transfer, 120 (2018) 9-17.

[26] G. Zhang, X. Zhang, D. Wang, Z. Jin, X. Qin, Performance evaluation and 600 operation optimization of the steam ejector based on modified model, Applied

[27] S. Dykas, M. Majkut, K. Smołka, M. Strozik, Comprehensive investigations into 603 1085.

606

607

608

609

610

611

612

613

614

[28] X. Han, W. Zeng, Z. Han, Investigating the dehumidification characteristics of the low-pressure stage with blade surface heating, Applied Thermal Engineering, (2019) 114538.

[29] Y. Yang, X. Zhu, Y. Yan, H. Ding, C. Wen, Performance of supersonic steam ejectors considering the nonequilibrium condensation phenomenon for efficient energy utilisation, Applied Energy, 242 (2019) 157-167.

[30] A. Kantrowitz, Nucleation in very rapid vapor expansions, The Journal of chemical physics, 19(9) (1951) 1097-1100.

[31] J. Young, The spontaneous condensation of steam in supersonic nozzle, Physico 
[32] ANSYS Fluent Theory Guide, ANSYS Inc., USA, (2017).

617 [33] F.R. Menter, Two-equation eddy-viscosity turbulence models for engineering applications, AIAA journal, 32(8) (1994) 1598-1605.

619

620

621

622

623

624

625

626

627

628

629

630

631

632

633

634

635

636

[34] G. Zhang, X. Zhang, F. Wang, D. Wang, Z. Jin, The relationship between the nucleation process and boundary conditions on non-equilibrium condensing flow based on the modified model, International Journal of Multiphase Flow, 114 (2019) 180-191.

[35] K. Ariafar, D. Buttsworth, G. Al-Doori, R. Malpress, Effect of mixing on the performance of wet steam ejectors, Energy, 93 (2015) 2030-2041.

[36] C. Wen, N. Karvounis, J.H. Walther, Y. Yan, Y. Feng, Y. Yang, An efficient approach to separate $\mathrm{CO} 2$ using supersonic flows for carbon capture and storage, Applied energy, 238 (2019) 311-319.

[37] A.J. Hedbäck, Theorie der spontanen Kondensation in Düsen und Turbinen, ETH Zurich, 1982.

[38] Y. Yang, J.H. Walther, Y. Yan, C. Wen, CFD modeling of condensation process of water vapor in supersonic flows, Applied Thermal Engineering, 115 (2017) 13571362.

[39] C.H. Kim, J.H. Park, D.I. Kim, J.H. Baek, Numerical analysis of non-equilibrium steam condensing flows in various Laval nozzles and cascades, Engineering Applications of Computational Fluid Mechanics, 11(1) (2017) 172-183.

[40] A.G. Gerber, M.J. Kermani, A pressure based Eulerian-Eulerian multi-phase 


\section{Nomenclature}

\section{Abbreviations}

CFD computational fluid dynamics

SST

shear stress transport

\section{Symbols}

$a$

coefficient for nozzle design

$C_{p} \quad$ constant pressure specific heat

$D$

diameter

$h_{l v}$

latent heat

$H$

total specific enthalpy

$J$

nucleation rate

$k_{B}$

Boltzmann's constant

$\mathrm{Kn}$

Knudsen number

$L_{1} \quad$ length of the converging part of the nozzle

$m_{v} \quad$ mass of a vapour molecule

$N \quad$ droplet number per volume

p pressure

Pr Prandtl number

$q_{c} \quad$ condensation coefficient

$r$

droplet radius 


\begin{tabular}{|c|c|}
\hline$r_{c}$ & critical droplet radius \\
\hline$R_{v}$ & gas constant \\
\hline$S$ & degree of supersaturation \\
\hline$t$ & time \\
\hline$T$ & temperature \\
\hline$u$ & velocity \\
\hline$x$ & Cartesian coordinates \\
\hline$Y$ & liquid fraction \\
\hline$\alpha$ & modelling parameter \\
\hline$\beta$ & modelling parameter \\
\hline$\gamma$ & specific heat ratio \\
\hline$\lambda_{e f f}$ & effective conductivity \\
\hline$\lambda_{v}$ & vapour conductivity \\
\hline$\lambda_{t}$ & turbulent thermal conductivity \\
\hline$v$ & modelling correction coefficient \\
\hline$\rho$ & density \\
\hline$\sigma$ & surface tension \\
\hline$\tau$ & viscous stress \\
\hline$\phi$ & Kantrowitz's correction factor \\
\hline$\Gamma$ & condensation mass rate \\
\hline \multicolumn{2}{|c|}{ Subscript } \\
\hline$i, j$ & Cartesian tensor notation \\
\hline
\end{tabular}




\begin{tabular}{|ll|}
\hline in & nozzle inlet \\
$l$ & liquid phase \\
$s$ & saturation condition \\
th & nozzle throat \\
$v$ & vapour phase \\
\hline
\end{tabular}

640 